\title{
Promise and pitfalls of g-ratio estimation with MRI
}

\author{
Jennifer S. W. Campbell ${ }^{\mathrm{a}, \mathrm{b}, *}$, Ilana R. Leppert ${ }^{\mathrm{a}}$, Sridar Narayanan ${ }^{\mathrm{a}}$, Mathieu Boudreau ${ }^{\mathrm{a}}$, Tanguy Duval ${ }^{\mathrm{b}}$, Julien Cohen-Adad ${ }^{\mathrm{b}, \mathrm{e}}$, \\ G. Bruce Pike ${ }^{c}$, Nikola Stikov ${ }^{\mathrm{b}, \mathrm{d}}$ \\ ${ }^{a}$ Montreal Neurological Institute, McGill University, Montreal, QC, Canada \\ ${ }^{b}$ NeuroPoly Lab, Institute of Biomedical Engineering, Polytechnique Montréal, Montréal, QC, Canada \\ ${ }^{c}$ University of Calgary, Calgary, $A B$, Canada \\ ${ }^{d}$ Montreal Heart Institute, Université de Montréal, Montréal, QC, Canada \\ ${ }^{e}$ Functional Neuroimaging Unit, CRIUGM, Université de Montréal, Montréal, QC, Canada
}

\section{Abstract}

The fiber g-ratio is the ratio of the inner to the outer diameter of the myelin sheath of a myelinated axon. It has a limited dynamic range in healthy white matter, as it is optimized for speed of signal conduction, cellular energetics, and spatial constraints. In vivo imaging of the g-ratio in health and disease would greatly increase our knowledge of the nervous system and our ability to diagnose, monitor, and treat disease. MRI based g-ratio imaging was first conceived in 2011, and expanded to be feasible in full brain white matter with preliminary results in 2013. This manuscript reviews the growing g-ratio imaging literature and speculates on future applications. It details the methodology for imaging the g-ratio with MRI, and describes the known pitfalls and challenges in doing so.

Keywords: g-ratio, MRI, myelin imaging, diffusion MRI, white matter, microstructure

\section{Introduction}

The g-ratio is an explicit quantitative measure of the relative myelin thickness of a myelinated axon, given by the ratio of the inner to the outer diameter of the myelin sheath. Both axon diameter and myelin thickness contribute to neuronal conduction velocity, and given the spatial constraints of the nervous system and cellular energetics, an optimal g-ratio of roughly 0.6-0.8 arises (Rushton, 1951; Waxman, 1975; Chomiak \& Hu, 2009). Spatial constraints are more stringent in the central nervous system (CNS), leading to higher g-ratios than in peripheral nerve (Chomiak \& Hu, 2009). Study of the g-ratio in vivo is interesting in the context of healthy development, aging, learning, and disease progression and treatment. In demyelinating diseases such as multiple sclerosis (MS), g-ratio changes and axon loss occur, and the g-ratio changes can then partially recover during the remyelination phase (Albert et al., 2007). The possibility that the g-ratio is dependent on gender during development, driven by testosterone differences, has recently been proposed (Paus \& Toro, 2009) and investigated (Pesaresi et al., 2015, Perrin et al. 2009). Possible clinical ramifications of a non-optimal g-ratio include "disconnection" syndromes such as schizophrenia (Paus \& Toro, 2009), in which g-ratio differences have been reported (Uranova et al. 2001; Du et al. 2014).

The g-ratio is expected to vary slightly in healthy neuronal tissue. The relationship between axon size and myelin sheath thickness is close to, but not exactly, linear (Berthold et al. 1983), with the nonlinearity more pronounced for larger axon size (Hildebrand \& Hahn, 1978), where the g-ratio is higher

*jennifer.campbell@mcgill.ca
(Graf von Keyserlingk \& Schramm, 1984). During development, axon growth outpaces myelination, resulting in a decreasing g-ratio as myelination catches up (Schröder et al., 1988). There is relatively little literature on the spatial variation of the g-ratio in healthy tissue. Values in the range 0.72-0.81 have been reported in the CNS of small animals (mouse, rat, guinea pig, rabbit) (Benninger et al., 2006: Duval et al., 2016a). Other primary pathology and disorders may lead to an abnormal gratio. These include leukodystrophies and axonal changes, such as axonal swelling in ischemia.

There are many outstanding questions in demyelinating disease that could be best answered by imaging the g-ratio in vivo. For example, in MS, disease progression is still the topic of active research. Most histopathological data are from patients at the latest stages of the disease. Therapies designed to promote remyelination are being developed to augment immunomodulatory and immunosuppressive treatments. Since remyelination of chronically demyelinated axons would be neuroprotective, this may help slow progression in MS. Detailed longitudinal study of the extent of remyelination can therefore aid in choosing avenues for therapy. While techniques exist for measurement of the g-ratio ex vivo (Graf von Keyserlingk \& Schramm, 1984), measurement of the g-ratio in vivo is an area of active research.

Many MR imaging contrasts are sensitive to changes in the g-ratio, in that they are sensitive to changes in, e.g., the total myelin content or total fiber content. The purpose of the g-ratio imaging formulation is to decouple the fiber density from the g-ratio, such that a more complete and specific picture of the microstructural detail can be achieved. In recent work (Stikov et al. 2011. 2015b), it has been shown that the combination of 
an MRI marker that is sensitive to the myelin volume fraction (MVF) and an MRI marker that is sensitive to the intra-axonal volume fraction or axon volume fraction (AVF) is sufficient to compute a g-ratio for each voxel, i.e., an aggregate g-ratio, without explicit estimation of axon diameter and myelin sheath thickness. The aggregate g-ratio is a function of the ratio of the MVF to the AVF. g-Ratio imaging does not involve the acquisition of a novel contrast, but is a specific computation using the parameters (MVF and AVF) extracted from existing contrasts. The challenge then becomes how to estimate the MVF and the AVF precisely and accurately with MRI. The g-ratio imaging framework, coupled with independent microstructural measures such as axon diameter (Assaf et al., 2008, Zhang et al. 2011), comprises the field of in vivo histology of white matter. The ultimate goal is to describe microstructure in detail on a scale much finer than an imaging voxel, as a distribution or single aggregate value for each the voxel.

\section{Methodology}

\subsection{The g-ratio formulation}

As previously defined, the g-ratio is the ratio of the inner to the outer diameter of the myelin sheath of a myelinated axon (see Fig. 1). It has been shown in recent work (Stikov et al. 2011, 2015b) that the aggregate g-ratio can be expressed as a function of the myelin volume fraction and the axon volume fraction, and hence can be estimated without explicit measurement of these diameters:

$$
g=\sqrt{\frac{1}{1+M V F / A V F}} .
$$

This formulation applies to any imaging modality (e.g., electron microscopy (EM) and scanning electron microscopy (SEM), where the MVF and AVF can be measured after segmentation of the image - see Fig 1, but it is of particular interest to be able to estimate the g-ratio in vivo. MRI provides us with several different contrast mechanisms for estimation of these volume fractions, and given $\mathrm{MVF}_{M R I}$ and $\mathrm{AVF}_{M R I}$, we can estimate $\mathrm{g}_{M R I}$. Hereon, we sometimes refer to $\mathrm{g}_{M R I}$ as "the g-ratio", but note that it is derived from MRI images with certain contrasts sensitive but not equivalent to the MVF and AVF. We forego the subscript MRI on the MVF and AVF acronyms, but it should be clear from context when these are MR estimates. Estimation of these quantities is discussed in the next sections.

\subsection{Axon volume fraction}

Diffusion MRI is particularly well suited to aid in the estimation of the axon volume fraction. As we will see later, it cannot estimate the absolute AVF, because there is very little signal from the myelin in a typical diffusion MRI experiment. However, the relative AVF can be estimated, because diffusion MRI is sensitive to the displacement distribution of water molecules moving randomly with thermal energy, and this displacement distribution is affected by the cellular structure present in the tissue. As the molecules impinge on the cellular membranes, organelles, and cytoskeleton, the displacement

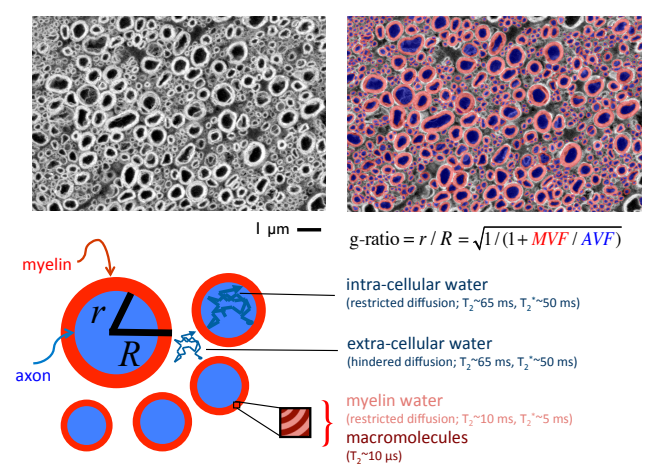

Figure 1: Original (top left) and segmented (top right) scanning electron micrograph showing axons of white matter, the intra-axonal space (blue), and the myelin (red). The segmentation was performed with AxonSeg (Zaimi et al. 2016). In the SEM image, the myelin appears white because of osmium preparation. The fiber g-ratio is the ratio of the inner to the outer radius of the myelin sheath surrounding an axon. The aggregate g-ratio can be expressed as a function of the myelin volume fraction (MVF) and the axon volume fraction (AVF). The myelin macromolecules, myelin water, and intra- and extra-axonal water compartments all have distinct properties, which can be exploited to generate MRI images from which the respective compartment volume fractions can be estimated.

distribution takes on a unique shape depending on the environment. Intra-axonal diffusion is said to be restricted, resembling free Gaussian diffusion at short diffusion times, but departing markedly from Gaussianity at longer times, where the displacement distribution is limited by the pore shape. There will be a sharp drop in the probability of displacement beyond the cell radius. Extra-axonal diffusion is said to be hindered, resembling free Gaussian diffusion, but with a smaller variance due to impingement of motion.

Many diffusion models exist for explicit estimation of the relative cellular compartment sizes. These include neurite orientation density and dispersion imaging (NODDI) (Zhang et al. 2012), the composite hindered and restricted model of diffusion (CHARMED) (Assaf \& Basser, 2005), diffusion basis spectrum imaging (DBSI) (Wang et al. 2011), restriction spectrum imaging (RSI) (White et al. 2013), white matter tract integrity (WMTI) from diffusion kurtosis imaging (DKI) (Fieremans et al., 2011), temporal diffusion spectroscopy (Xu et al., 2014), microscopic anisotropy obtainable from multiple pulsed field gradient MRI (Shemesh et al., 2012; Zhou et al., 2013; Avram et al., 2013), the spherical mean technique (Kaden et al. 2016), the distribution of anisotropic microstructural environments in diffusion-compartment imaging (DIAMOND) (Scherrer et al. 2016), and many others (Rokem et al. 2015, Stanisz et al. 1997). It is also possible to perform NODDI with relaxed constraints (NODDIDA) (Jelescu et al. 2016a), and to do this calculation analytically (LEMONADE) (Novikov et al., 2016). 
Another approach, termed the apparent fiber density (AFD), uses high diffusion weighting to virtually eliminate the hindered diffusion signal, leaving only intra-axonal water (Raffelt et al., 2012). It has been used to estimate the relative axon volume fraction of different fiber populations in a voxel. A modification, termed the tensor fiber density (TFD), can be performed with lower diffusion weighting (Reisert et al. 2013).

The simplest diffusion MRI models do not differentiate between the tissue compartments. For instance, the diffusion tensor (Basser et al. 1994) models the entire displacement distribution as an anisotropic Gaussian function. The parameters defining this function will change if the intra-axonal volume fraction changes, but to what extent is it practical to extract meaningful quantitative compartment volume fractions from the tensor? Recently, a framework called NODDI-DTI has been developed, in which the proximity of DTI-based parameters to the computed NODDI parameters is assessed (Edwards et al. 2016), given certain assumptions. The FA and the mean diffusivity (MD) are highly correlated in straight, parallel fiber bundles, and will change with changing AVF, leading to estimates of the relative intra-axonal volume. However, this formulation is probably an oversimplification of the microstructural situation, and more detailed modeling is a better choice to ensure specificity to white matter fibers.

All of the diffusion models above are potential candidates for use in g-ratio imaging. They have strengths and weaknessess that will not be detailed here. The original full-brain $\mathrm{g}_{\text {MRI }}$ demonstration (Stikov et al. 2015b) employed the NODDI model of diffusion. It was chosen because of its suitability in the presence of complex subvoxel fiber geometry, including fiber divergence, which may occur to a significant scale in almost all imaging voxels (Ghosh et al., 2016), and its suitability on clinical scanners with relatively low gradient strength. Having a fast implementation of the model fitting with numerical stability is important for large studies, hence, the convexoptimized AMICO implementation is beneficial (Daducci et al., 2015).

While diffusion MRI is a modality of choice for imaging microstructure, it can only measure the displacement distribution of water molecules that are visible in a diffusion MRI experiment. This limits us to water that is visible at an echo time (TE) on the order of 50-100 ms, and therefore excludes water that is trapped between the myelin bilayers, which has a $T_{2}$ on the order of $10 \mathrm{~ms}$. Hence, the estimates provided by these models are of the intra-axonal volume fraction of the diffusion visible volume. Myelin does not figure in the models. Given, e.g., the NODDI model outputs, a complementary myelin imaging technique must be used to estimate the absolute axon volume fraction. The AVF is given by

$$
A V F=(1-M V F)\left(1-v_{i s o}\right) v_{i c},
$$

where $v_{i s o}$ and $v_{i c}$ are the isotropic and restricted volume fractions from the NODDI model, and the MVF is obtained from one of many possible myelin mapping techniques, examples of which are discussed below.

Diffusion contrast may not be our only window onto the axon volume fraction. Recent work has shown that it is possible to disambiguate the myelin, intra-axonal, and extra-axonal water compartments using complex gradient echo (GRE) images (Sati et al., 2013, Wu et al., 2016). The myelin water is separable from the combined intra- and extra-axonal water using multicomponent $\mathrm{T}_{2}^{*}$ reconstruction, providing a myelin marker (see below). However, incorporation of the phase of the GRE images potentially allows us to separate all three compartments based on frequency shifts. Challenges include the fact that the frequency shift is dependent on the orientation of the axon to the main magnetic field $\mathrm{B}_{0}$. When the axon is oriented perpendicular to $\mathrm{B}_{0}$, the myelin water will experience a positive frequency shift, the intra-axonal water a negative frequency shift, and the extra-axonal water will not experience a frequency shift.

Note that the AVF as defined by these diffusion MRI models is specific to white matter. While it makes sense to define an axon volume fraction in grey matter, the models in general cannot distinguish between axons and dendrites. The NODDI model's $v_{i c}$ parameter, for example, is "neurite density", i.e., the density of all cellular processes that can be assumed to have infinitely restricted diffusion in their transverse plane. Hence, $\mathrm{g}_{M R I}$ from such MRI data is undefined in grey matter.

\subsection{Fiber volume fraction}

The fiber volume fraction (FVF), or fiber density, is the sum of the AVF and the MVF. Can diffusion MRI, or any other MRI contrast mechanism, measure the total fiber volume fraction itself? Clearly, GRE images have potential, as discussed above. Is diffusion imaging sensitive to the FVF, as opposed to the AVF? While myelin water is virtually invisible in diffusion MRI, diffusion MRI is not insensitive to myelin. First, the ratio of the intra- to extra-axonal diffusion MRI visible water in a voxel will change as the myelin volume fraction in that voxel changes. In early work on the fiber g-ratio, it was shown that assuming a simple white matter model of straight, parallel cylinders, the fractional anisotropy (FA) of the diffusion tensor, which weighs both intra-axonal and extra-axonal anisotropy, is proportional to the total fiber volume fraction, with a quadratic relationship (Stikov et al., 2011). The NODDI parameter $v_{i c}$ also changes with demyelination, even if all axons remain intact. Second, diffusion acquisitions are heavily $\mathrm{T}_{2}$ weighted, and $\mathrm{T}_{2}$ is myelin-sensitive. The total diffusion weighted signal thus decreases as myelin content increases. However, to robustly quantify myelin volume fraction, it is necessary to add a second contrast mechanism, even if it is additional $\mathrm{T}_{2}$ weighted images, to the scanning protocol. This is discussed below.

Despite the nomenclature, as noted above, even the Apparent Fiber Density and Tensor Fiber Density are in fact relative axon densities. They would provide a relative FVF only if the g-ratio is constant. In a recent study of the g-ratio (Mohammadi et al. 2015), the TFD was equated with the FVF, not the AVF, for input to the g-ratio formula. The g-ratio is a function of the ratio of the MVF to the AVF (Eq. 1), or alternately, the ratio of the MVF to the FVF:

$$
g=\sqrt{1-M V F / F V F}
$$


This means that conclusions reached about the variation of the g-ratio found by equating the TFD with the FVF will be robust in the above case. Absolute g-ratios in the above case were calibrated to have a mean of 0.7 in healthy white matter.

We note that if diffusion MRI were capable of estimating the absolute FVF (or AVF) as well as the ratio of the intraaxonal to extra-fiber water, the g-ratio could immediately be estimated from these two quantities, without further myelin imaging. This has yet to be done robustly, and it is therefore preferable to use a more robust independent myelin marker.

\subsection{Myelin volume fraction}

There are many different contrasts and computed parameters that are sensitive to myelin (Laule et al., 2007). The possible sources of signal from the myelin compartment are the ultrashort $\mathrm{T}_{2}$ protons in the macromolecules of the myelin sheath itself $\left(\mathrm{T}_{2} \sim 10 \mu \mathrm{s}\right)$ and the short $\mathrm{T}_{2}$ water protons present between the phospholipid bilayers $\left(\mathrm{T}_{2} \sim 10 \mathrm{~ms}\right.$, see Fig. 1). Most MRI contrast mechanisms are sensitive to myelin content, but few are specific, for reasons that are detailed in section 3.3 . The myelin phospholipid bilayers create local Larmor frequency variations for water protons in their vicinity due to diamagnetic susceptibility effects. This results in myelin content modulated transverse relaxation times $\mathrm{T}_{2}^{*}$ (Hwang et al., 2010) and $\mathrm{T}_{2}$, and longitudinal relaxation time $\mathrm{T}_{1}$. It has been shown that macromolecular content is the dominant source of variance in $T_{1}$ in the brain (Rooney et al., 2007). The local Larmor field shift (fL) and the susceptibility itself $(\chi)$ can be computed as well (Liu et al., 2015). Ultra-short TE (UTE) imaging can be used to image the protons tightly bound to macromolecules (Wilhelm et al. 2012, Du et al., 2014). An alternate approach to isolating the myelin compartment is magnetization transfer (MT) imaging, where the ultra-short $\mathrm{T}_{2}$ macromolecular proton pool size can be estimated by transfer of magnetization to the observable water pool.

MT based parameters sensitive to macromolecular protons include the magnetization transfer ratio (MTR) (Wolff et al. 1991), the MT saturation index $\left(\mathrm{MT}_{\text {sat }}\right)$ (Helms et al. 2008. 2010), the macromolecular pool size (F) from quantitative magnetization transfer (Sled \& Pike, 2001, Yarnykh, 2002, Ramani et al., 2002), single-point two-pool modeling (Yarnykh 2012), and inhomogeneous MT (Varma et al., 2015).

Alternately, the myelin water can be imaged with quantitative multicomponent $\mathrm{T}_{2}$ (MacKay et al., 1994) or $\mathrm{T}_{2}^{*}(\overline{\mathrm{Du}}$ et al., 2007; Alonso-Ortiz et al., 2016) relaxation, which yields the myelin water fraction (MWF) surrogate for myelin density. Variants include gradient and spin echo (GRASE) MWF imaging (Does \& Gore, 2000; Prasloski et al., 2012), linear combination myelin imaging (Jones et al., 2004; Vidarsson et al., 2005), $\mathrm{T}_{2}$ prepared MWF imaging (Oh et al., 2006), multi-component driven equilibrium single point estimation of $\mathrm{T}_{2}$ (mcDESPOT) (Deoni et al., 2008), direct visualization of the short transverse relaxation time component via an inversion recovery preparation to reduce long $\mathrm{T}_{1}$ signal (ViSTa) (Oh et al., 2013), and a fast adiabatic $\mathrm{T}_{2}$-prep and spiral readout approach (FAST-T2) (Nguyen et al. 2016). Other alternate approaches exploiting myelin-modulated relaxation times include combined contrast imaging $\left(\mathrm{T}_{1 W} / \mathrm{T}_{2 W}\right)$ (Glasser \& Van Essen, 2011) or independent component analysis (Mangeat et al., 2015). Proton density is also sensitive to macromolecular content, and the protondensity based macromolecular tissue volume (MTV) (Mezer et al. 2013) has been used as a quantitative myelin marker.

While these MRI measures have been shown to correlate highly with myelin content (Thiessen et al. 2013; Schmierer et al., 2007; Gareau et al., 2000, Laule et al., 2006, Mottershead et al. 2003), they have not been incorporated in a specific tissue model in a manner similar to the diffusion signal, and hence some calibration is needed. This is still a topic of research. Caveats of improper calibration of the MVF are discussed in section 3.3

Above, we have discussed imaging techniques for both diffusionvisible microstructure and myelin. Any multi-modal modal imaging protocol with contrasts such as these, sensitive to the axon and myelin volume fractions, is sensitive to the g-ratio (e.g., (Molina-Romero et al., 2016; Nossin-Manor et al., 2015, De Santis et al., 2016a; Bells et al. 2011)). The purpose of the explicit g-ratio formulation is to create a measure that is specific to the g-ratio. It provides us with a metric that is independent of the fiber density, which none of the MRI contrasts sensitive to the MVF and AVF separately accomplish. It is interesting to ask whether we could use a technique such as deep learning (Bengio, 2009) to estimate the g-ratio, skipping explicit modeling completely.

\subsection{MRI acquisition protocol: an example}

Given the large number of MRI contrasts that could potentially be used in the g-ratio imaging framework, we cannot prescribe an exact protocol for g-ratio imaging here. However, in the following sections, we illustrate several important points about g-ratio imaging using experimental data acquired at our site. The following describes the acquisition protocol we have used.

We acquired data from healthy volunteers and from multiple sclerosis patients. These data were acquired on a Siemens $3 \mathrm{~T}$ Trio MRI scanner with a 32 channel head coil. A $\mathrm{T}_{1 W}$ structural MPRAGE volume with $1 \mathrm{~mm}$ isotropic voxel size was acquired for all subjects. For diffusion imaging, the voxel size was $2 \mathrm{~mm}$ isotropic. For most experiments, the NODDI diffusion protocol consisted of $7 \mathrm{~b}=0 \mathrm{~s} / \mathrm{mm}^{2}, 30 \mathrm{~b}=700 \mathrm{~s} / \mathrm{mm}^{2}$, and $64 \mathrm{~b}=2000 \mathrm{~s} / \mathrm{mm}^{2}$ images, 3x slice acceleration, 2x GRAPPA acceleration, all acquired twice with AP-PA phase encode reversal. For the other experiments, as detailed below when they are introduced, the slice acceleration and phase encode reversal were not employed. For a dataset optimized for diffusion tensor reconstruction, a dataset with 99 diffusion encoding directions at $b=1000 \mathrm{~s} / \mathrm{mm}^{2}$ and $9 \mathrm{~b}=0 \mathrm{~s} / \mathrm{mm}^{2}$ images was acquired.

For magnetization transfer images, we also used $2 \mathrm{~mm}$ isotropic voxels to match the diffusion imaging voxel size. For MTR, one 3D non-selective PD-weighted RF-spoiled gradient echo (SPGR) scan was acquired with $\mathrm{TR}=30 \mathrm{~ms}$ and excitation flip angle $\alpha=5^{\circ}$, and one MT-weighted scan was acquired 
with the same parameters and an MT pulse with $2.2 \mathrm{kHz}$ frequency offset and $540^{\circ} \mathrm{MT}$ pulse flip angle. For $\mathrm{MT}_{\text {sat }}$ computation, these same MT-on and MT-off scans were used, with one additional $\mathrm{T}_{1}$-weighted scan with $\mathrm{TR}=11 \mathrm{~ms}$ and excitation flip angle $\alpha=15^{\circ}$. For qMT computation, 10-point logarithmic sampling of the z-spectrum from $0.433-17.235 \mathrm{kHz}$ frequency offset was acquired, with two MT pulse flip angles for each point, $426^{\circ}$ and $142^{\circ}$, and excitation flip angle $\alpha=4.5^{\circ}$. The qMT acquisition was accelerated with $2 x$ GRAPPA acceleration. Additional scans for correction of the maps included $\mathrm{B}_{1}$ field mapping using the double angle technique (Boudreau et al., 2017, Stollberger \& Wach, 1996), with $60^{\circ}$ and $120^{\circ}$ flip angles, $\mathrm{B}_{0}$ field mapping using the two-point phase difference technique, with $\mathrm{TE}_{1} / \mathrm{TE}_{2}=4.0 / 8.48 \mathrm{~ms}$, and $\mathrm{T}_{1}$ mapping using the variable flip angle technique (Fram et al., 1987), with flip angles $3^{\circ}$ and $20^{\circ}$. Additional $\mathrm{T}_{2}$-FLAIR and $\mathrm{PD}_{W}$ images were acquired for the MS subjects to aid in lesion segmentation.

\section{The pitfalls: outstanding technical challenges}

In this section, we discuss pitfalls and outstanding issues in g-ratio imaging. These include the challenge of combining multiple different contrast mechanisms, limitations in diffusion modeling, specificity and calibration of myelin markers, and the limitation of estimating a single g-ratio metric in the presence of subvoxel heterogeneity. Experimental results are included in these sections to illustrate these problems. In the experiments, we focus on the NODDI and DTI models and MT-based myelin imaging because they were used in the early g-ratio imaging publications (Stikov et al., 2011,2015b), but many of these concerns apply to any chosen AVF and MVF markers.

\subsection{Confounds in multi-modal image acquisition}

The computation of the g-ratio metric includes several preprocessing steps, including distortion and field inhomogeneity correction, that deserve further discussion. The MT-based contrasts are acquired with spin-warp acquisition trains, and the diffusion-based contrasts are acquired with single-shot EPI. When any acquisition details are changed, the distortions in the images change, and co-registration of voxels for voxelwise quantitative computations becomes more difficult.

The blip-up blip-down phase encode strategy (section 2.5) allows for precise correction of susceptibility-induced distortion in the diffusion images (Andersson et al. 2003). Lack of correction for this distortion leads to visible bands of artifactually high $\mathrm{g}_{M R I}$ near tissue-CSF interfaces (see, e.g, (Campbell et al., 2014, Cercignani et al., 2016a)). This was illustrated by Mohammadi et al. (Mohammadi et al., 2015) (see Fig. 2). Uncorrected diffusion MRI data leads to g-ratios in the vicinity of unity at the edge of the genu of the corpus callosum, caused by voxels where the AVF is artifactually high (containing little or no CSF), and the MVF low (because the correctly localized voxels actually contain CSF). The white matter - CSF boundary is a region of obvious misregistration, but much of the frontal lobe suffers from susceptibility induced distortion, and would therefore have incorrect g-ratios.

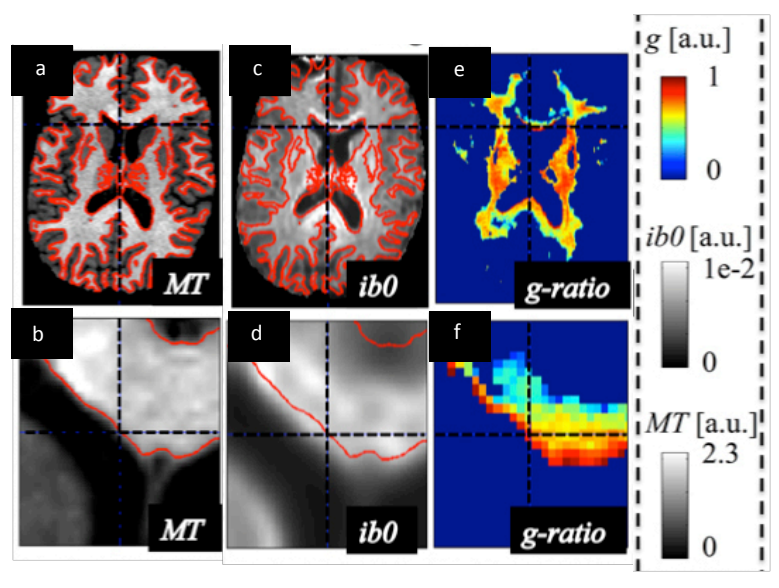

Figure 2: Misregistration artifact due to susceptibility-induced distortion in diffusion weighted images. At left is an $\mathrm{MT}_{\text {sat }}$ image with white matter outlined in red, for one slice (a) and a cropped region at the genu (b). In the center (c,d) is an original EPI diffusion scan with no diffusion weighting and contrast inverted (ib0). The misregistration with the $\mathrm{MT}_{\text {sat }}$-defined white matter boundary is marked. At right $(\mathrm{e}, \mathrm{f})$ is the g-ratio computed with these contrasts. Uncorrected diffusion MRI data leads to g-ratios in the vicinity of unity at the edge of the genu, caused by voxels where the AVF is artifactually high (containing little or no CSF), and the MVF low (because the correctly localized voxels actually contain CSF). Reproduced from (Mohammadi et al. 2015).

Multi-modal imaging protocols are a powerful tool for investigation of microstructure. We have thus far discussed combining multiple images with partially orthogonal contrasts in order to estimate the g-ratio. However, problems such as the above misregistration issue arise. Can a single acquisition train provide multiple contrasts? One such approach was described recently for simultaneous mapping of myelin content and diffusion parameters (De Santis et al. 2016a). It consists of an inversion-recovery preparation before a diffusion weighted sequence, allowing for fitting of a model that incorporates both $\mathrm{T}_{1}$ (a myelin marker (Stüber et al., 2014)) and axonal attributes. This approach is conceptually extensible to other myelin-sensitive preparations or modifications of a diffusion weighted sequence, such as quantitative $\mathrm{T}_{2}$ estimation (Kim et al., 2016) or MT preparation (Gupta et al. 2003).

Can the g-ratio be estimated using a single contrast mechanism? This could also offer inherent co-registration, as well as possibly increasing the acquisition speed. As discussed in section 2.2, analysis of complex GRE images may lead us to a technique for estimating the MVF and AVF volume fractions from one set of images.

Making a multi-modal imaging protocol short enough for the study of patient populations and use in the clinic is a considerable challenge. In section 3.3. we investigate the MT saturation (Helms et al., 2008) as a more time efficient replacement for qMT. There exist other short MT-based approaches, such as single-point two-pool modeling (Yarnykh, 2012) and inhomo- 
geneous MT (Varma et al., 2015). Another approach could be to use compressed sensing (Lustig et al., 2007) for MT-based acquisitions (McLean et al., 2017). Recent advances in myelin water fraction imaging (Nguyen et al., 2016) may make MWF estimation more efficient, and GRE-based myelin water fraction approaches (Alonso-Ortiz et al. 2016) may also offer a faster approach for estimating the MWF, with the possibility, as mentioned above, to eliminate the diffusion imaging part of the protocol.

Diffusion imaging has benefited from many acceleration approaches in recent years, including parallel imaging, which can also be used in the myelin mapping protocols, slice multiplexing (Setsompop et al., 2012), and hardware advances such as the CONNECTOM gradient system.

\subsection{Diffusion modeling}

\subsubsection{Model parameters}

The diffusion MRI post-processing techniques described in section 2.2 give a range of outputs. Some are physical quantities (such as the diffusion displacement distribution; kurtosis), while some are parameters of detailed biological models (such as the intra-axonal volume fraction). Models are valuable, but the user has to be aware of the assumptions made.

The parameter space in existing models ranges from three free parameters in NODDI to six (Assaf \& Basser, 2005), twenty three (Jespersen et al., 2010), and thirty one (Novikov et al., 2016, Jelescu et al. 2015) in other models, with the maximum dependent on acquisition details. Recent analysis hypothesizes that the lower number of free parameters in, e.g., NODDI and CHARMED, may be matched to the level of complexity possible on current clinical systems (Ferizi et al., 2015), while high gradient strength, high b-values, and more b-shells may be necessary for more complex models (Jelescu et al. 2015), and would make them more optimal. This is a general problem with multi-exponential models when diffusion weighting is weak (Kiselev \& Il'yasov, 2007). On standard MR systems, relaxing the constraints on fixed parameters has been shown to lead to degeneracy of solutions (Jelescu et al., 2016a). Regularization approaches such as the spherical mean technique (SMT) (Kaden et al. 2016) have been employed in an attempt to make the problem less ill-posed.

One of the fixed parameters in the NODDI model is the parallel diffusivity in the intra- and extra-axonal space, both set to the same fixed value. Other models explicitly model these as unequal; for instance, WMTI assumes that the intra-axonal diffusivity is less than or equal to the extra-axonal diffusivity. The actual values are unknown, however simulations have shown that the assumption of equal parallel diffusivities leads to a 34$53 \%$ overestimation of the intra-axonal compartment size if the diffusivities are in fact unequal (Jelescu et al., 2015), with the intra-axonal diffusivity either greater than or less than the extraaxonal diffusivity. If the fixed diffusivities are incorrect, the intra-axonal compartment size estimated by the NODDI model will be non-zero even if there is no anisotropy (Lampinen et al. 2017).

Independent of whether the intra- and extra-axonal parallel diffusivities are equal, another source of this bias is the tor- tuosity model (Szafer et al., 1995) employed by many models, including NODDI, DIAMOND, and the SMT. This model computes the perpendicular extra-axonal diffusivity as a function of the diffusion-visible intra-axonal volume fraction of the nonCSF tissue ( $\mathrm{v}_{i c}$ in the NODDI model). This tortuosity estimate is bound to be inaccurate because the tortuosity is expected to vary as the absolute fiber volume fraction of the non-CSF tissue, not the diffusion-visible fiber volume fraction (Jelescu et al. 2015). These two quantities are very different, as the myelin and axon volume fractions are almost equal in healthy tissue (Stikov et al. 2015b). The MVF could be explicitly included in the equation, and would be expected to result in a myelin volume dependent reduction in $v_{i c}$. However, in healthy tissue, where the FVF should scale roughly as the $\mathrm{v}_{i c}$ parameter, the tortuosity model of Szafer $e$ t al. does not appear to hold when applied to experimental data with independent estimates of the parallel and perpendicular extra-axonal diffusivities (Novikov et al. 2016). The model is probably not correct for varying packing density on the sub-voxel scale (Novikov et al., 2011); it has been shown to depend on the packing arrangement and breaks down for tight axon packing (Fieremans et al., 2008, Novikov \& Fieremans, 2012). This might explain the discrepancy between model and experiment mentioned above, because the geometry of axonal packing can vary considerably for a given average volume fraction.

Another fixed parameter in the NODDI model is the $\mathrm{T}_{2}$ relaxation time of all tissue, assumed to be the same, even in CSF. This leads to an overestimation of $\mathrm{v}_{i s o}$, which can be corrected (Bouyagoub et al. 2016) given $\mathrm{T}_{2}$ estimates from, e.g., a $\mathrm{T}_{2}$ mapping technique such as mcDESPOT (Deoni et al., 2008).

\subsubsection{Geometry in diffusion imaging}

Diffusion MRI is exquisitely sensitive to fiber geometry. The fractional anisotropy may be more sensitive to geometry than to any microstructural feature (Hutchinson et al., 2016). Hence, microstructural models must be careful to take geometry (crossing, splaying, curving, microscopic packing configuration) into account. A typical diffusion imaging voxel is roughly $8 \mathrm{~mm}^{3}$, while the axons probed by microstructural models are on the order of one micron.

Early work on the fiber g-ratio investigated the human corpus callosum, where it was assumed that the white matter fibers were effectively straight and parallel (Stikov et al., 2011). The use of this model that assumes straight, parallel fibers suffers from several problems. First, the regions of the brain where this model can be expected to hold at all are very limited, as there are crossing or splaying fibers in up to $95 \%$ of diffusion MRI voxels in parenchyma (Jeurissen et al., 2010, Behrens et al., 2007: Ghosh et al., 2016), and curvature is almost ubiquitous at standard imaging resolution. Even the axons of callosal fibers are not straight and parallel, with splay up to $18^{\circ}$ (Ronen et al. 2014; Mollink et al., 2016). Second, the model assumes a relatively uniform, if random, packing of axons on the scale of the MRI voxel. Due to the nonlinear nature of the FA, it will depend strongly on the packing geometry. If two voxels, one with densely packed axons and one with sparsely packed axons, are combined into one, the FA for that voxel will be less 

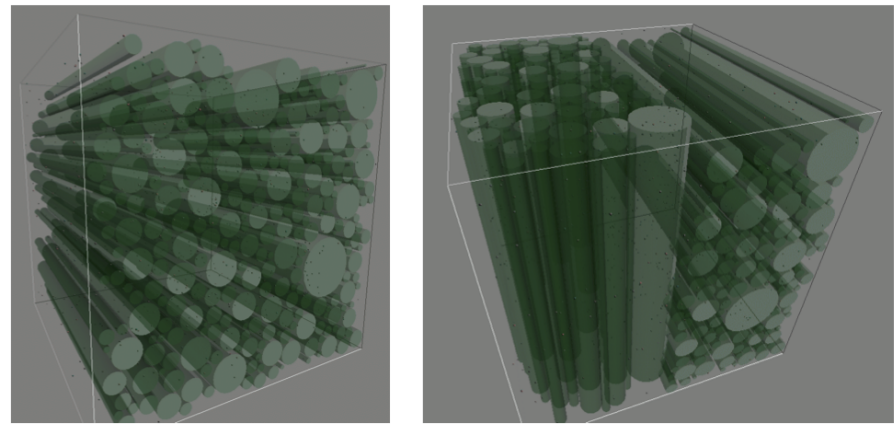

Figure 3: Simulated fibers in straight, parallel configuration (left) vs. crossing (right), with equal fiber volume fraction and similar distributions of axon diameter and position. The NODDI model underestimates the FVF by $3.8 \pm 0.3 \%$ in the crossing fiber case, whereas the DTI model underestimates the FVF by $58.7 \pm 3.2 \%$.

than the average of the two original voxels, whereas the fiber density will be the average of the fiber densities. Third, FA is in practice acquisition and b-value dependent.

\subsubsection{Experiments: AVF from NODDI in the presence of crossing fibers}

The NODDI model that has been used in several g-ratio imaging studies to date assumes there is a single fiber population with potential splay or curvature, but does not explicitly model crossings. To what extent does the fiber dispersion model of NODDI handle crossing fiber bundles? We have employed the diffusion MRI simulator dSim (Sveinsson \& Dougherty, 2011) to investigate this question (Campbell et al., 2014). We simulated realistic axonal packing (Aboitiz et al., 1992) in voxels with straight, parallel fibers and with two equal size bundles of straight fibers crossing at $90^{\circ}$ (See Fig 3). Fiber volume fractions were set equal for both configurations and were varied from 0.3 to 0.7 . g-Ratios were varied from 0.7 to 0.9 . The diffusion weighted signal was generated, and the NODDI model parameters computed using the NODDI Matlab toolbox (NODDI Matlab Toolbox, 2013). The FVF was computed from the NODDI parameters using the known MVF. The computed FVF was $3.8 \pm 0.3 \%$ lower in the crossing fiber case for the NODDI-based FVF. This demonstrates that the NODDI model, while not explicitly designed for crossing fibers, gives acceptable results in this case, and can be used for full-brain g-ratio estimation at standard voxel size with significant subvoxel fiber crossing, with only a small decrease in the estimated FVF due to partial volume averaging of fiber orientations.

\subsubsection{Experiments: Comparison of DTI and NODDI for FVF estimation}

NODDI works optimally with diffusion MRI measurements made on at least two shells in q-space, i.e., two different nonzero b-values, although recent work has proposed solutions for single shell data, at least where certain assumptions can be made about the tissue, or where high b-values are used (Grussu et al. 2014, Magnollay et al. 2014). In contrast, the diffusion tensor can be robustly fitted and the fiber volume fraction inferred (Stikov et al. 2011) (see section 2.3) using a much more sparsely sampled, single shell dataset. Many research programs have large databases of single-shell diffusion data, often with limited angular sampling of q-space as well. It is therefore of interest to explore to what extent such data, using the diffusion tensor model, can be used in investigation of the g-ratio.

In the simulations described above, we also computed the diffusion tensor using in-house software (mincdiffusion 2013). The FVF $\left(\mathrm{FVF}_{D T I}\right)$ was computed from the FA using the quadratic relationship determined from previous simulations (Stikov et al., 2011). As expected, the FA is not a predictor of FVF in the presence of crossing fibers: the computed DTI-based FVF was $58.7 \pm 3.2 \%$ lower in the crossing fiber case compared to the parallel fiber case.

To compare NODDI and DTI in vivo, diffusion and qMT data were acquired as described in section 2.5 for one healthy volunteer, without slice acceleration or phase encode reversal. The qMT data were processed with in-house software and the NODDI parameters as described above. Additionally, the diffusion tensor was calculated using the $b=1000 \mathrm{~s} / \mathrm{mm}^{2}$ diffusion shell. The AVF, MVF, and $\mathrm{g}_{M R I}$ were computed voxelwise from the diffusion and qMT data as described in section 2.1. the NODDI-based FVF $\left(\mathrm{FVF}_{N O D D I}\right)$ is the sum of the MVF and the AVF computed from Eq. 2, and $\mathrm{FVF}_{D T I}$ was calculated from the fractional anisotropy of the diffusion tensor using the quadratic relationship (Stikov et al., 2011). The corpus callosum was skeletonized on the FA image and a voxel-wise correlation between the FVF computed from DTI and from NODDI and qMT was performed for these voxels. The coefficient of proportionality between $\mathrm{F}$ and MVF was determined from previous EM histological analysis (Stikov et al., 2015b).

Fig. 4 shows the FVF computed using both NODDI and the FA from DTI in the skeleton of the healthy human corpus callosum. The Pearson correlation coefficient between the FVF measured using the two techniques was $\mathrm{r}=0.79$, with $\mathrm{FVF}_{\mathrm{NODDI}}=$ $1.15 * \mathrm{FVF}_{\mathrm{DTI}}+0.00$. This indicates a slight discrepancy between the FVF using NODDI compared to DTI, and a reasonably high correlation between techniques on the skeleton.

Possible explanations for the higher estimates using NODDI appear in section 3.2.1, although without ground truth is is difficult to say which approach is more accurate. Additionally, because the FA does not explicitly model compartments, it is subject to partial volume effects. While partial volume averaging with CSF will decrease the FA, the FA-based quadratic FVF model appears to break down in this case. This effect could possibly be reduced by applying the free water elimination technique (Pasternak et al. 2009) to obtain the correct FVF for the non-CSF compartment and then scaling to reflect the partial volume averaging with CSF afterward. To conclude, while the FA is generally a poor indicator of FVF, it may be a reasonable surrogate in certain special cases when data are limited.

It is interesting to consider how useful imaging a crosssection of a white matter fascicle may be, regardless of the model used. If the g-ratio can be assumed to be constant along an axon, measurement of a cross-section is useful. However, in many pathological situations, such as Wallerian degeneration, it is of interest to study the entire length of the axon. 


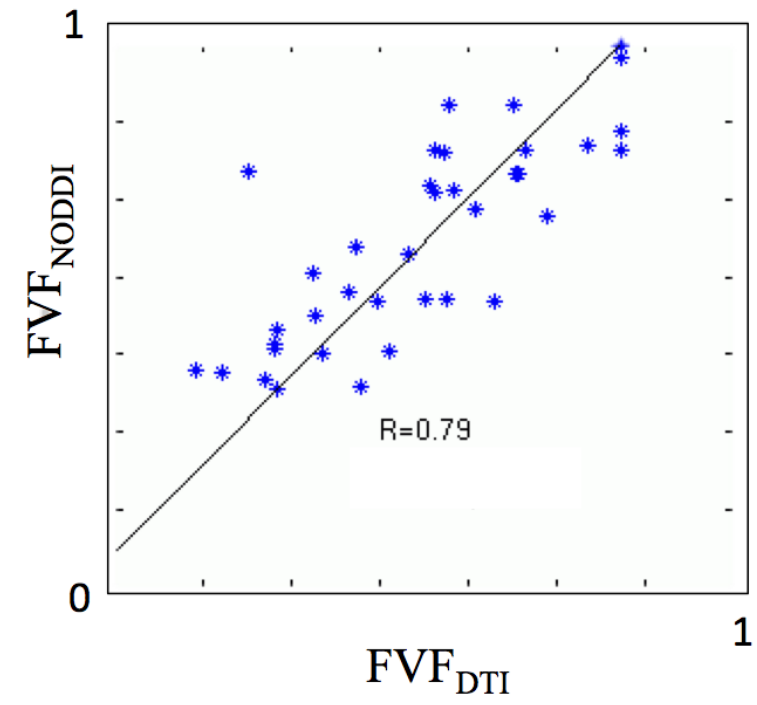

Figure 4: Correlation between DTI- and NODDI-derived fiber volume fraction on the skeleton of the corpus callosum. $\mathrm{FVF}_{D T I}$ was calculated from the fractional anisotropy of the diffusion tensor using a quadratic relationship (Stikov et al. 2011, and $\mathrm{FVF}_{N O D D I}$ is the sum of the MVF from qMT and the AVF computed from Eq. 2

\subsubsection{Restricted extracellular diffusion}

Most existing diffusion models assume that extra-axonal diffusion is Gaussian, hindered by the structures present, but not restricted. However, observation of tightly packed axons in microscopy (e.g., Fig 1] indicates that the intra- and extra-axonal spaces may not be as distinguishable as the models assume. It is unclear to what extent the extra-axonal diffusion is nonGaussian. If axons are packed tightly together, is extra-axonal diffusion non-Gaussian? It is not clear whether the water mobility through the tight passageways between fibers is distinguishable from the restricted diffusion within spaces surrounded by contiguous myelin. If signal from the extra-axonal space is erroneously attributed to the intra-axonal space, the model output will be incorrect.

Some models make no attempt to distinguish between intraand extra-cellular restricted diffusion, meaning the pore size estimates may reflect a mixture of the two (Ning et al. 2016). Time-dependent (i.e., non-Gaussian) diffusion has recently been observed in the extra-axonal space using long (Fieremans et al. 2016, De Santis et al. 2016b) and short (Xu et al., 2014) diffusion times. This may be due to axon varicosity, axonal beading, or variation in axonal packing (Fieremans et al., 2016). In order for the intra- and extra-axonal compartment fractions to be estimated, the diffusion MRI signal from these compartments must be distinguishable with the experimental paradigm and modeling used.

Diffusion modeling is an active field, and advances in the near future will hopefully improve precision and accuracy of AVF estimates using diffusion MRI. Histological validation may aid in understanding the strengths and limitations of these estimates. At present, the limitations of these models propagate to the g-ratio, as do the limitations of MVF estimates, which are discussed below.

\subsection{MVF calibration}

In this section, we investigate the effect of imperfect calibration of MRI markers of the MVF. As an example, we present detailed experimental analysis of the magnetization transfer ratio (MTR), which is a commonly used myelin marker that is implemented on clinical scanners and would be a logical first choice for g-ratio imaging in the clinic. Computing a precise and accurate MVF from the MTR is, however, challenging, as is detailed below. We also investigate the performance of $\mathrm{MT}_{\text {sat }}$ (Helms et al. 2008) as a potential time-efficient replacement for qMT in the estimation of the MVF. Care must be taken in the use of any myelin marker in quantitative g-ratio computation.

How do we make a quantitative estimate of the MVF from myelin sensitive MRI markers? Linear correlations have been shown between the individual myelin sensitive metrics (such as F (Thiessen et al., 2013, Schmierer et al., 2007), MTR (Gareau et al. 2000, Schmierer et al., 2004), $\mathrm{R}_{1}$ (Mottershead et al., 2003), MWF (Laule et al., 2006), and MTV (Duval et al., 2016b)) with the MVF from histology. Given the linear correlations that have been established, a logical first approximation is to assume a linear relationship between the chosen myelin-sensitive metric and the MVF. Then, using the macromolecular pool size $\mathrm{F}$ as an example, the relationship is

$$
M V F=c F+b,
$$

with $c$ and $b$ constants. While a non-zero value for $b$ has been indicated by some studies (Thiessen et al., 2013, West et al. 2017), this may be an artifact due to the inherent bias in linear regression. The assumption of a linear relationship hinges on the assumption that non-myelin macromolecular content scales linearly with myelin content, but this relationship can break down in disease, or even in healthy tissue. In disease, astrocyte scarring, glial cell processes, and inflammatory cell swelling could all modulate the relationship between a marker of macromolecular content and the MVF. If the myelin and non-myelin macromolecular content do scale linearly, as is assumed here, a theoretical prior that $b=0$ is reasonable. However, $b$ could reflect a fixed population of macromolecules that are uncorrelated with myelin content. It would most likely be negative, i.e., $\mathrm{F}$ could be positive with $\mathrm{MVF}=0$.

There is evidence that even if a simple scaling relationship exists between $\mathrm{F}$ and MVF, it is dependent on acquisition and post-processing details. For instance, a recent study calibrated $\mathrm{F}$ at two different sites, and found a different scaling factor for each (Cercignani et al., 2016a). These scaling factors in turn differ from those obtained from other investigations (Stikov et al. 2015b; Dula et al., 2010; Thiessen et al. 2013). Hence, careful calibration for each study must be performed. Several studies have calibrated scaling factors based on a given expected g-ratio in healthy white matter (Cercignani 
et al. 2016a, Mohammadi et al. 2015). However, the g-ratio in healthy white matter is not precisely known.

None of the myelin-sensitive MRI markers is $100 \%$ specific to myelin, and most are sensitive to myelin in a slightly different way. Magnetization transfer contrast is specific to macromolecules, and more specific to lipids than to proteins (Kucharczyk et al., 1994). Macromolecules in the axon membrane itself, in neurofilaments within the axons, and in glial cell bodies, will contribute to the MT signal, with myelin constituting only $50 \%$ of the macromolecular content in healthy white matter (Bjarnason et al., 2005). Additionally, MT-based metrics such as the magnetization transfer ratio will have residual contrast from other mechanisms. We expect the MTR contrast to vary linearly with macromolecular content, but also with $\mathrm{T}_{1}$ (Vavasour et al. 2011). $\mathrm{T}_{1}$ has the opposite sensitivity to myelin than does the MT effect (Mottershead et al. 2003), meaning that these effects work against each other, reducing the dynamic range and power of MTR as a marker of myelin. Furthermore, $\mathrm{T}_{1}$ is sensitive to iron and calcium content, intercompartmental exchange, and diffusion, and hence sensitive to axon size (Harkins et al., 2016) and axon count (Schmierer et al., 2008). This means the relationship between MTR and MVF may not be monotonic, and is certainly nonlinear. This nonlinearity is evident in published plots of MTR vs. F, e.g., that shown by Levesque et al. (Levesque et al., 2005), and the lack of dynamic range of MTR is also evident (Levesque et al., 2005. Garcia et al., 2012). The $\mathrm{MT}_{\text {sat }}$ technique aims to remove the $\mathrm{T}_{1}$ dependence in MTR. Both MTR and $\mathrm{MT}_{\text {sat }}$ depend on the offset frequency used in the acquisition. ihMT shows promise as a more myelin-specific MT marker due to its sensitivity to specific molecules in myelin that broaden the z-spectrum asymmetrically, although it has recently been shown that asymmetric broadening is not essential to generate a non-zero ihMT signal (Manning et al. 2016), and the technique suffers from low signal. qMT is the most comprehensive of the MT-based myelin markers, although its use is impeded by long acquisition times, and its parameters appear to be sensitive to the specific model and fitting algorithm.

Proton-density based techniques (Mezer et al., 2013) will, like MT, be sensitive to all macromolecules, with a different weighting on these macromolecules compared to the lipid dominated MT signal. Relaxation-based myelin markers are also not $100 \%$ specific to myelin. The confounds with using $\mathrm{T}_{1}$ directly were mentioned above, and the dependence on iron and calcium concentration, intercompartmental exchange and diffusion will also affect $\mathrm{T}_{2}$. $\mathrm{T}_{2}^{*}$ is also sensitive to iron concentration, as well as fiber orientation (Cohen-Adad, 2014). Isolating the short $\mathrm{T}_{2}$ or short $\mathrm{T}_{2}^{*}$ compartment enhances specificity to myelin, but MWF estimates vary nonlinearly with myelin content as the sheath thins and exchange and diffusion properties are modulated (Levesque \& Pike, 2009, West et al., 2014, Harkins et al. 2012). Variants may suffer from reduced accuracy or precision, for example, the mcDESPOT technique has been shown to overestimate the MWF (Bouhrara et al., 2016) and lack precision (Lankford \& Does, 2013). Combining $\mathrm{T}_{1}$ and $\mathrm{T}_{2}$ in various ways (Glasser \& Van Essen, 2011; Mangeat et al. 2015) may increase specificity, although this approach relies on myelin being the dominant source of contrast. In the UTE technique, it is as yet unclear how to map the signal directly to myelin content. In addition to these confounds, most of these myelin markers have recently been shown to have orientation dependence. These include $\mathrm{T}_{2}^{*}, \chi$ (Rudko et al., 2014, Liu et al., 2015), and $\mathrm{T}_{2}$ of the macromolecular pool (Pampel et al., 2015).

While these myelin imaging techniques are certainly powerful tools in the study of healthy and diseased brain, can they be used reliably in the g-ratio imaging framework? As an illustration of the effects of miscalibration of myelin markers, consider the following scenario (Campbell et al., 2016). We investigate three MT-based myelin markers: MTR, $\mathrm{MT}_{\text {sat }}$, and macromolecular pool size F. We assume a simple linear scaling between our MRI marker and the MVF. As described in previous work (Stikov et al. 2015b), we calibrate F using combined in vivo MRI acquisition ex vivo electron microscopy in the macaque. We then calibrate MTR and $\mathrm{MT}_{\text {sat }}$ to match the mean F-based MVF in white matter. We subsequently compute $\mathrm{g}_{M R I}$, using the NODDI model of diffusion and the MVF derived from the myelin markers (Eq.s 2 1).

\subsubsection{MVF calibration: Experimental Methods}

MTR, $\mathrm{MT}_{\text {sat }}, \mathrm{qMT}$, and NODDI data were acquired for five healthy volunteers and one MS patient, as described in section 2.5. Additionally, for two of the healthy subjects, we acquired one MT-on image with an offset frequency of $1.2 \mathrm{kHz}$, which is the standard MTR sequence used at our site. For the MS patient, the $\mathrm{MT}_{\text {sat }}$ images were computed from the qMT MT-off and MT-on (MT pulse offset $2.732 \mathrm{kHz}$, flip angle $142^{\circ}$ ) images and one additional $\mathrm{T}_{1 W}$ image with $\mathrm{TE}=3.3 \mathrm{~ms}, \mathrm{TR}=15 \mathrm{~ms}$, and excitation flip angle $\alpha=20^{\circ}$. The diffusion images were preprocessed using FSL (Smith et al., 2004), and the NODDI parameters were computed using the NODDI matlab toolbox (NODDI Matlab Toolbox, 2013). The qMT computation of the macromolecular pool size $F$ was performed using in-house software (Sled \& Pike, 2001, Cabana et al., 2015), including $\mathrm{B}_{0}$ and $\mathrm{B}_{1}$ correction. $\mathrm{MT}_{\text {sat }}$ was computed according to Helms et al. (Helms et al., 2008, 2010), using the $2.2 \mathrm{kHz}$ offset frequency data, and also the $1.2 \mathrm{kHz}$ offset frequency data where available. MTR was computed for both offset frequencies where available. A semi-empirical $\mathrm{B}_{1}$ correction was made (Weiskopf et al. 2013) to correct for higher order $\mathrm{B}_{1}$ effects. Binary segmentation of white and grey matter was performed using an in-house pipeline, using the MPRAGE image only. Lesion segmentation for the MS subject was performed with in-house software.

The combined MRI/histology dataset (Stikov et al., 2015b) was used to scale each myelin marker (MTR, $\mathrm{MT}_{\text {sat }}$, and F) to give the MVF, with the assumption of a linear relationship (Eq. 4) with intercept $b=0$. Correlations between the three myelin markers were computed in brain parenchyma. Percent differences were computed between healthy white matter and healthy grey matter for each of the three myelin markers. The AVF was computed using Eq. 2, and g-ratios were computed in the MS and healthy brains using Eq. 11. Average g-ratios were computed in healthy white matter, normal appearing white matter 

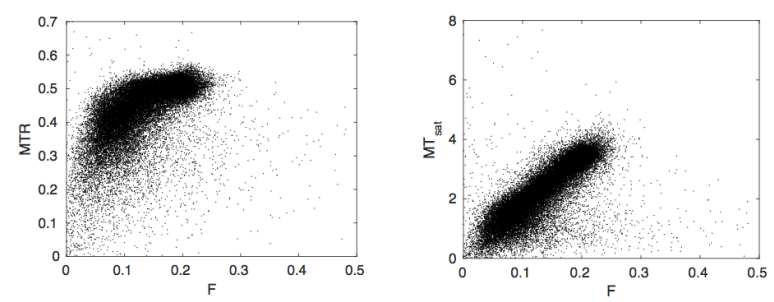

Figure 5: MTR plotted versus $\mathrm{F}$ (left) and $\mathrm{MT}_{\text {sat }}$ plotted versus $\mathrm{F}$ (right) in parenchyma for one subject at $2.2 \mathrm{kHz}$ offset frequency. The MTR vs. F plot shows a marked nonlinearity ( $r=0.62$ over five subjects; $r=0.57$ for the subject shown in this plot), as is expected. $\mathrm{MT}_{\text {sat }}$ increases the linearity of the relationship ( $r=0.80$ over five subjects; $r=0.78$ for the subject shown in this plot) and the dynamic range.

\section{(NAWM), and MS lesions.}

A theoretical computation was also performed, varying the mapping of an arbitrary myelin metric to MVF using Eq. 4. We separately varied the slope (c) and the intercept (b) for a range of fiber volume fraction values and mapped the computed gratio as a function of FVF. When varying the slope, the intercept was fixed at the origin.

\subsubsection{MVF calibration: Results}

For the $2.2 \mathrm{kHz}$ offset frequency (Helms et al., 2008), the average correlation of MTR with $\mathrm{F}$ was $\mathrm{r}=0.62(\mathrm{p}<0.001)$, and of $\mathrm{MT}_{\text {sat }}$ with $\mathrm{F}$ was $\mathrm{r}=0.80(\mathrm{p}<0.001)$ in parenchyma. The relationship between $\mathrm{MT}_{\text {sat }}$ and $\mathrm{F}$ is more linear than the relationship between MTR and $\mathrm{F}(\mathrm{p}<0.0001)$. Fig. 5 shows plots of MTR versus $\mathrm{F}$ (left) and $\mathrm{MT}_{\text {sat }}$ versus $\mathrm{F}$ (right) in parenchyma for one subject at $2.2 \mathrm{kHz}$ offset frequency. Of note, the plot of MTR versus $F$ has a distinctive nonlinear shape, similar to that seen in the literature (Levesque et al., 2005). When $\mathrm{T}_{1}$ effects are reduced using $\mathrm{MT}_{\text {sat }}$, the linearity and dynamic range increase. For the two subjects in which the lower, $1.2 \mathrm{kHz}$ offset frequency was also used to compute both MTR and $\mathrm{MT}_{\text {sat }}$, the average correlation of MTR with F was 0.55 and of $\mathrm{MT}_{\text {sat }}$ with F was 0.73 .

In healthy brain, the percent difference between white and grey matter was $15.02 \%$ for MTR, $40.08 \%$ for MT $_{\text {sat }}$, and $45.86 \%$ for $\mathrm{F}$. The narrower dynamic range of the MVF derived from MTR can also be seen in Fig. 6, where grey matter has markedly higher values. If this simple scaling to obtain the MVF is used in the g-ratio formula, the g-ratio in healthy white matter is relatively constant. However, when lesions exist, the contrast using the different MVF markers is very different. In the MS patient,

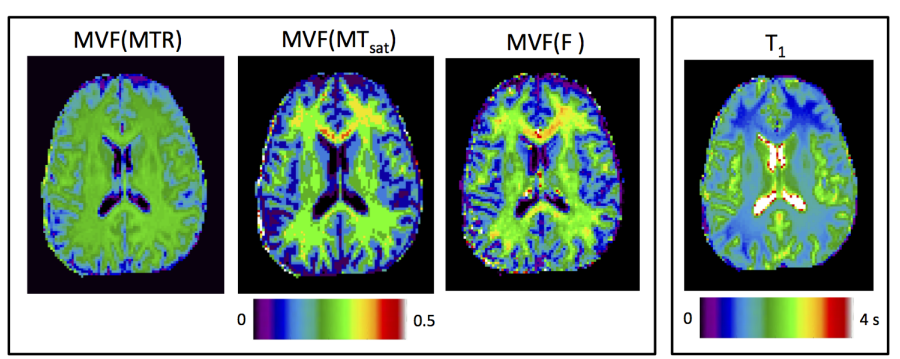

Figure 6: Left: Plots of the MVF derived from (from left to right) MTR, $\mathrm{MT}_{\text {sat }}$, and $\mathrm{F}$, in healthy brain. Right: The $\mathrm{MT}_{\text {sat }}$ calculation estimates a map of the apparent $\mathrm{T}_{1}$ and removes the $\mathrm{T}_{1}$ effects from the MTR map. $\mathrm{T}_{1}$ has the opposite contrast than does magnetization transfer, so these two effects work against each other in the MTR map, and the $\mathrm{MT}_{\text {sat }}$ map therefore has more contrast and a greater dynamic range. The inhomogeniety visible in the $\mathrm{T}_{1}$ map is due to $\mathrm{B}_{1}$ inhomogeniety, which is largely cancelled out in the $\mathrm{MT}_{\text {sat }}$ computation (Helms et al. 2008), although further correction for residual $\mathrm{B}_{1}$ effects is often performed (Weiskopf et al. 2013).
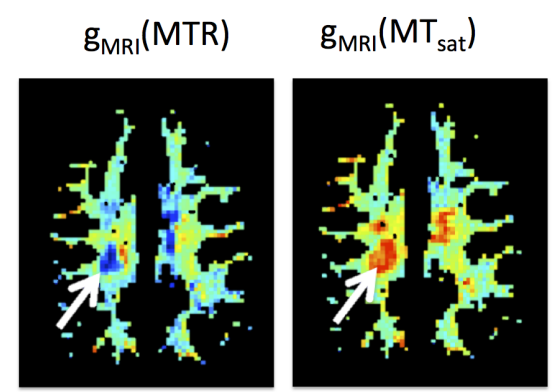
$\mathrm{g}_{\mathrm{MRI}}(\mathrm{F})$

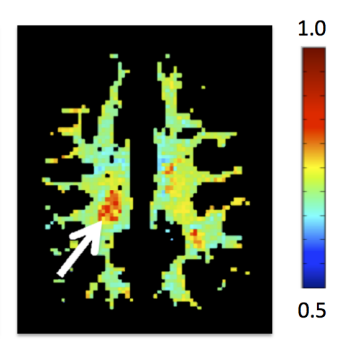

Figure 7: Plots of $\mathrm{g}_{M R I}$ computed using (from left to right) MTR, $\mathrm{MT}_{\text {sat }}$, and F, in the MS patient. The arrow indicates a lesion in which the apparent g-ratio is lower than in NAWM when using MTR, but higher than in NAWM when using $\mathrm{MT}_{\text {sat }}$ and $\mathrm{F}$.

the mean g-ratio in normal appearing white matter (NAWM) was 0.76 for all three MVF markers. In MS lesions, the mean g-ratio was $0.65,0.80$, and 0.80 , for MTR, $\mathrm{MT}_{\text {sat }}$, and F, respectively. Fig. 7 shows the spatial distribution of g-ratios in the MS patient for the three MVF markers.

Fig. 8 shows the theoretical effect of having an improper slope (top) or intercept (bottom) in the relationship between an arbitrary myelin marker and the MVF, in the case where the (theoretical) relationship is in fact linear. The plots show that the computed g-ratio becomes fiber density dependent, in addition to being incorrect.

\subsubsection{MVF calibration: Discussion}

The MTR is a commonly used myelin marker, however, due to $\mathrm{T}_{1}$ sensitivity, it lacks dynamic range. This results in unrealistic g-ratios in MS lesions that are lower than in NAWM. $\mathrm{T}_{1}$ is one of the possible MR-based myelin markers, but in the context of the MTR experiment, it confounds the contrast, because the MT effect dominates but is diminished by the $\mathrm{T}_{1}$ con- 

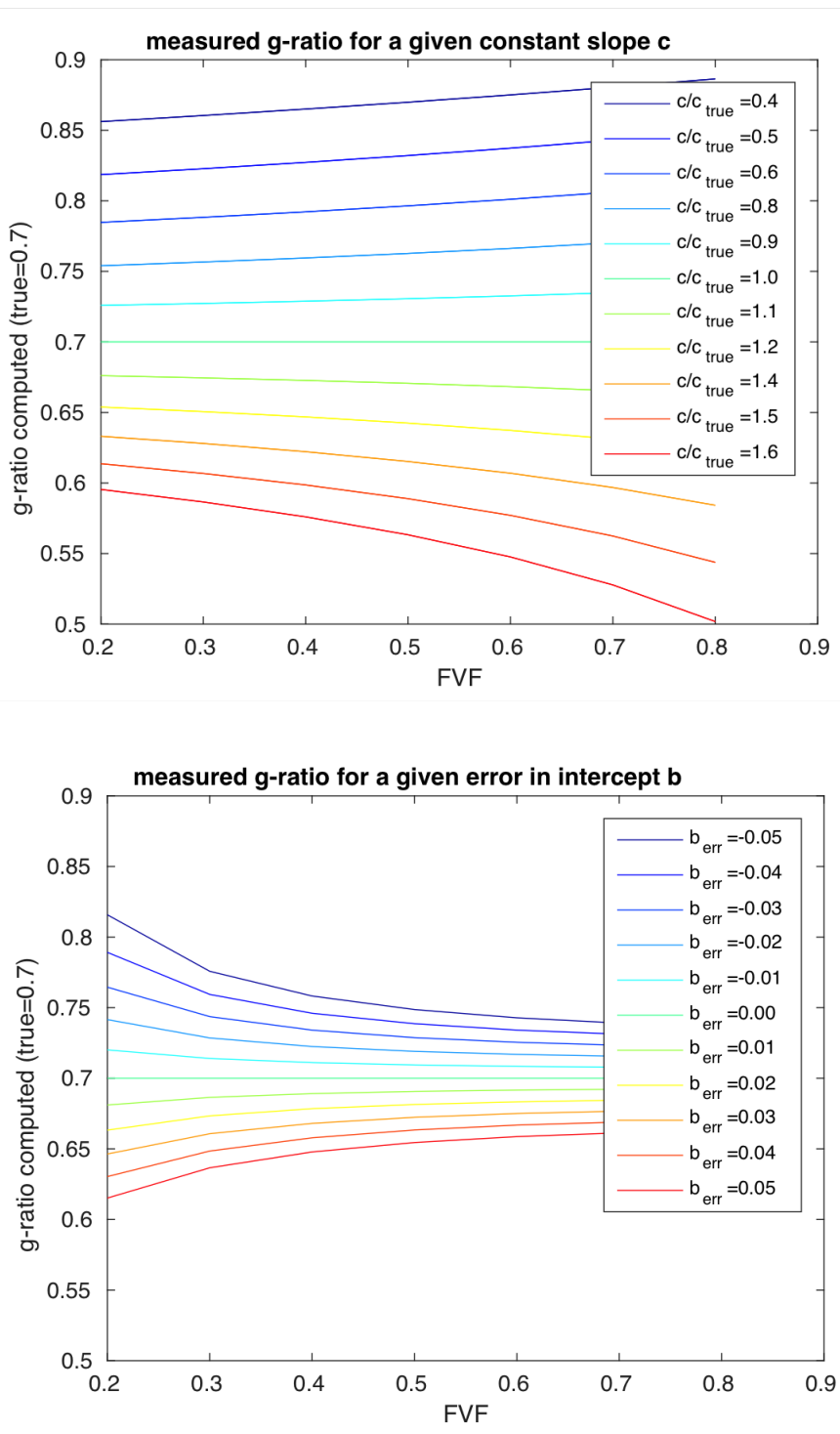

Figure 8: Effect of having an improper slope (top) or intercept (bottom) in the relationship between an arbitrary myelin marker and the MVF, in the case where the (theoretical) relationship is in fact linear. The plots show that the computed g-ratio becomes fiber density dependent, in addition to being incorrect. trast, which works against it. $\mathrm{MT}_{\text {sat }}$ correlates more highly with $\mathrm{F}$, which is obtained from an explicit qMT model designed to isolate the macromolecular tissue content. It is important to note, however, that this correlation may be driven to some extent by the different $B_{1}$ sensitivities of the techniques as the MTR was not corrected for $\mathrm{B}_{1}$ induced variability (Volz et al. 2010, Yarnykh \& Khodanovich, 2015). Independent of this demonstration of the potential of $\mathrm{MT}_{\text {sat }}$ for myelin mapping, researchers have found that $\mathrm{MT}_{\text {sat }}$ may be more sensitive to tissue damage than MTR in multiple sclerosis, with higher correlation with disability metrics (Lema et al., 2017). MT $_{\text {sat }}$ has recently been used by other groups in g-ratio imaging of healthy adults (Mohammadi et al., 2015).

If the MVF is miscalibrated in this g-ratio imaging formulation, there will be a residual dependence on fiber volume fraction in our formulation. This reduces the power of the g-ratio metric, which ideally is completely decoupled from the fiber density. Independent of specificity of the myelin marker, if the myelin calibration is inaccurate, this residual dependence on fiber volume fraction occurs. It is clear that the g-ratio metric we will compute is g-ratio weighted, and the better the calibration, the more weighted to the g-ratio it will be. Until quantitative myelin mapping is accurate, the g-ratio metric will not be specific to the g-ratio.

There is evidence that fiber density drops precipitously in some MS lesions (Stikov et al., 2015b). In Fig. 7, this was the case, and we can see that MTR does not drop enough, making MS lesions appear to have a lowered g-ratio instead of a higher g-ratio as expected. Inspecting the bottom (red) curve in Fig. 8. we see that even if there is a linear relationship between the myelin marker of choice and the MVF, miscalibration leads to an apparent g-ratio metric that is elevated in regions of lower fiber density, and significantly lower in regions of healthy fiber density. This occurs when in fact all of the fibers have the same g-ratio, and could easily be interpreted as hypomyelination in an MS subject or population.

As noted above, the problem of miscalibration of the myelin marker exists independent of the specificity of the myelin marker. However, specificity is itself a major confound, as previously detailed. In the case of demyelinating disease, one must also consider that, with many myelin markers, all myelin will affect the MR signal, even if it is not part of an intact fiber. Research indicates that in MS, there is acute demyelination followed by a period of clearance of myelin debris, followed by effective remyelination. During clearance, remyelination can occur, but this myelin is of poor quality (Lampron et al. 2015). On the scale of an MRI voxel, there can be myelin debris, poor remyelination, and higher quality remyelination. The extent to which myelin debris affects the myelin volume estimates may depend on the myelin mapping technique chosen. It is also not clear how well the estimates of MVF, and also AVF, behave at very low fiber density.

One possible solution for MVF calibration is to calibrate the g-ratio to a known value in certain regions of interest (Mohammadi et al. 2015, Cercignani et al., 2016a), as mentioned above. However, care must be taken that this step is not adjusting for differences in the diffusion part of the pipeline (e.g., 
different implementations of the diffusion model (Cercignani et al., 2016a)), and therefore still leaving a fiber density dependence. Additionally, the correct value in these regions of interest must be known. Calibration based on expected MVF would remove this sensitivity, but is subject to error due to partial volume averaging of white matter with other tissue. If the relationship between the myelin-sensitive metric and the MVF is not a simple scaling, such calibration will fail. Particular care needs to be taken when studying disease.

If the assumed relationship between the myelin marker and the MVF is incorrect, the computed g-ratio will be incorrect. Is it possible to compute a g-ratio that is correct to within a scaling factor, and not sensitive to the fiber density? This would require that the AVF or FVF be estimated independent of the MVF. Simple models such as the diffusion tensor, apparent fiber density (Raffelt et al. 2012), and tensor fiber density (Reisert et al. 2013), are indicators of fiber or axon density, but detailed modeling is most likely superior. Consideration of contrasts other than diffusion MRI, such as gradient-echo based approaches (Sati et al. 2013), might also help with this problem. The gratio is a function of the ratio of the MVF to the AVF, and a technique that measures this ratio directly would be optimal. However, due to the extremely short $\mathrm{T}_{2}^{*}$ of myelin, GRE based estimates would be of the myelin and axon water fraction, and hence would still need to be calibrated using the volumetric occupancy of water in these tissues.

In summary, both specificity and accuracy are important for both AVF and MVF estimation. For both AVF and MVF estimation, more sophisticated models may be required. For example, we have thus far ignored cell membranes. The axon membrane should technically be included in the AVF, and its volume is up to $4 \%$ of the AVF (Sepehrband et al., 2015), but it would most likely be included in the MVF using MT-based MVF estimation. It is also important to keep in mind the different sensitivities of different MRI markers (e.g., MWF from relaxometry vs. MT-based parameters), because these will not be expected to give identical $\mathrm{g}_{M R I}$ metrics. The determination of which MRI markers are most optimal for g-ratio imaging is still the topic of active research (Ellerbrock \& Mohammadi, 2017).

\section{4. g-Ratio distribution}

The g-ratio imaging paradigm extracts a single g-ratio metric per voxel. At typical imaging resolution feasible for the constituent MR images, a voxel contains hundreds of thousands of axons. As with the axon diameter, the g-ratio really applies to an individual axon, and takes on a broad distribution of values in tissue (Graf von Keyserlingk \& Schramm, 1984) (see Fig. 9, which shows the g-ratio distribution in the macaque corpus callosum, measured using electron microscopy). The range of myelination includes some unmyelinated axons within healthy white matter. The g-ratio distribution may broaden and become bi-modal in disease. Even within a single axon with intact myelin, the g-ratio may vary due to organelle swelling. Fiber bundles that cross within one voxel may have different g-ratio distributions. In development, some fibers within one fiber bundle will fully develop, while others will be pruned,
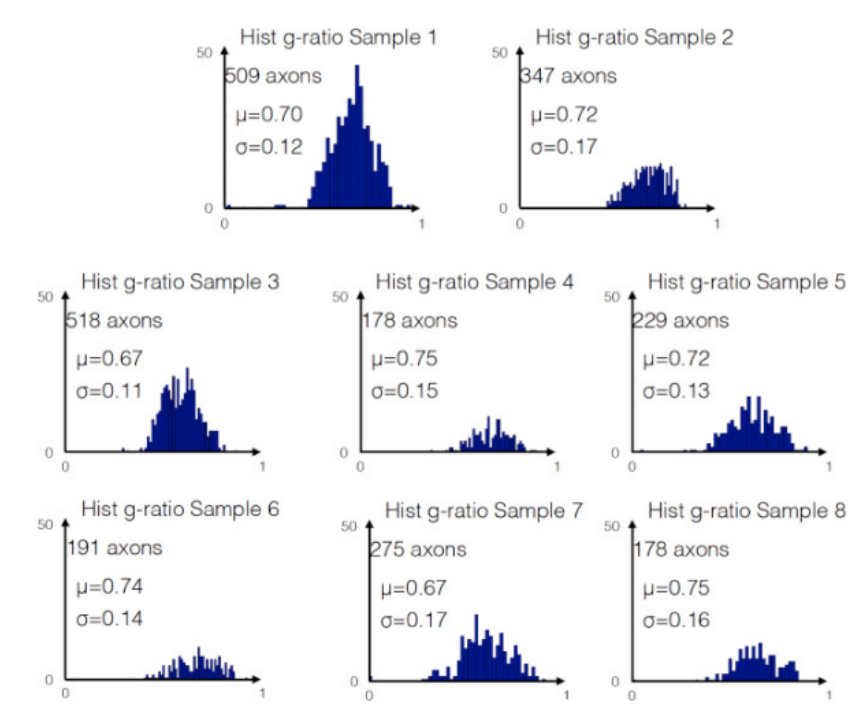

Figure 9: g-Ratio distributions from electron microscopy of the cynamolgus macaque corpus callosum, samples 1-8 from genu to splenium. Reproduced from (Stikov et al. 2015a).

resulting in an interim bimodal g-ratio distribution within the fascicle. The current MRI-based g-ratio framework will not be able to distinguish these cases, as it reports only an intermediate g-ratio value. It is robust to crossing fibers, in that it will report the same intermediate g-ratio value whether the separate bundles cross or lie parallel to each other. The broad g-ratio distribution is in part a resolution problem, but the g-ratio is expected to be heterogeneous on a scale smaller than we can hope to resolve with MRI.

The aggregate g-ratio we compute in the case of a distribution of values is not precisely fiber- or axon-area weighted, but is close to axon area weighted within a reasonable range of values (West et al. 2016b). Larger axons will have a greater weight in the aggregate g-ratio metric we measure. Simply put, the aggregate g-ratio is the g-ratio one would measure if all axons had the same g-ratio.

In the case of an ambiguous g-ratio distribution, what techniques can we use to infer what situation is occurring? In multiple sclerosis, for example, two possible scenarios probably occur frequently. One is patchy demyelination, on a scale much smaller than a voxel and smaller than the diffusion distance, and the other is more extensively and uniformly distributed thin myelin. These two scenarios could give rise to equal AVF, MVF, and aggregate g-ratio measurements. One possible way to differentiate these cases could be to look more closely at parameters available to us from diffusion models. It has been shown that the extra-axonal perpendicular diffusivity is relatively unchanged by patchy demyelination in a demyelinating mouse model (Jelescu et al., 2016b), because diffusing molecules encounter normal hindrance to motion on most of their trajectory, whereas the axon water fraction is sensitive to this patchy demyelination. Hence, the discrepancy between these two mea- 
sures can be taken as a measure of patchy demyelination. Alternatively, one can scan subjects longitudinally and infer disease progression. From the ambiguous timepoint described above, the axons in the patches that are demyelinated may die, leaving a decreased AVF and MVF, and a return to a near-healthy g-ratio. In the case of uniformly thin myelin, the remyelination may continue, leaving a near-healthy AVF, MVF, and gratio. Note that the g-ratio metric still does not distinguish these pathologically distinct cases. There are two unknowns - the fiber density and the g-ratio (or, alternately, the MVF and the AVF), and one must consider both to have a full picture of the tissue. Looking at the time courses, one can hypothesize what the g-ratio distribution was at the first timepoint.

It would be technically challenging to measure the g-ratio distribution in vivo. Even with an estimate of a distribution of diffusion properties, and an estimate of the distribution of a myelin-sensitive metric, the g-ratio distribution is ill-defined. However, several recent acquisition strategies may help us get closer to this aim. One approach is to take advantage of the distinguishable diffusion signal between different fiber orientations. In the IR-prepared diffusion acquisition described above (De Santis et al. 2016a), the model specifies multiple fiber populations with distinct orientations, each with its own $\mathrm{T}_{1}$ value. This means the diffusion properties, including the restricted pool fraction (a marker of intra-axonal signal from the CHARMED model), are paired with a corresponding $\mathrm{T}_{1}$ for each fiber orientation. Hence, a g-ratio metric could be computed for each fiber orientation. This could be of benefit in, e.g., microstructure informed white matter fiber tractography (e.g., (Girard et al. 2015)) of fiber populations with distinct g-ratios. "Jumping" from one fiber population to another is very common in tractography (Campbell \& Pike, 2013, Descoteaux et al., 2016, MaierHein et al., 2016), and constraining tractography to pathways with consistent microstructural features could help reduce false positives in regions of closely intermingling tract systems.

It may be possible, conceptually, to estimate the g-ratio distribution via a 2D diffusion-relaxation spectroscopic approach. While extremely acquisition intensive, 2D spectroscopy of $\mathrm{T}_{2}$ and the diffusion coefficient (Callaghan et al., 2003) has been demonstrated recently as a probe of microstructure (Kim et al. 2016). The acquisition involves making all diffusion measurements at different echo times. If a distribution of a myelin volume sensitive metric (here, $\mathrm{T}_{2}$ ) can be estimated simultaneously in 2D with a distribution of a diffusion-based metric sensitive to the axon volume, it may be possible to infer the distribution of g-ratios.

This has been an incomplete but useful list of pitfalls. Now, we will consider the promise of imaging the aggregate g-ratio weighted metric, despite its pitfalls. g-Ratio imaging is being explored in many different contexts, described below.

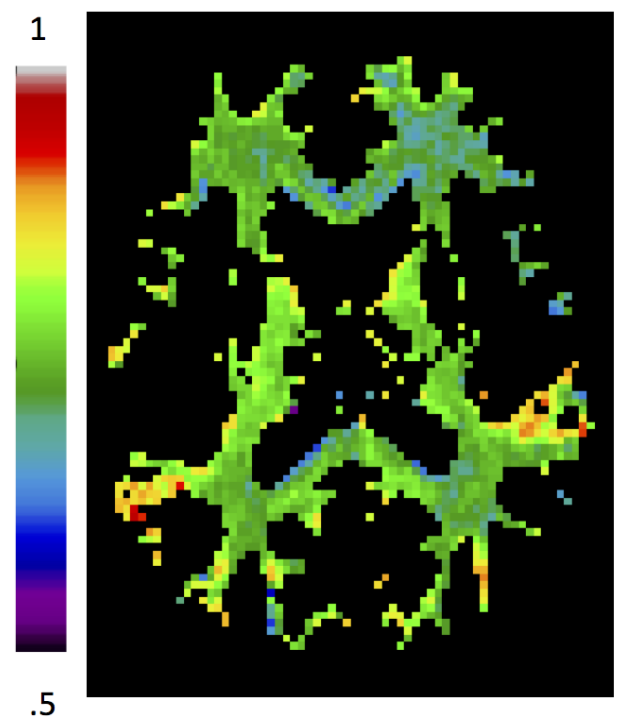

Figure 10: $\mathrm{g}_{M R I}$ in healthy white matter, imaged using qMT and NODDI.

\section{The promise: g-ratio imaging studies}

The promise of g-ratio imaging is its potential to provide us with valuable in vivo estimates of relative myelination. In the last few years, studies showing the potential of this framework have begun to emerge.

\subsection{Healthy white matter}

Fig. 10 shows an image of $\mathrm{g}_{M R I}$ in healthy white matter using our qMT and NODDI g-ratio protocol (see section 2.5). With our protocol, in healthy subjects, the $\mathrm{g}_{M R I}$ map is relatively flat, with a mean $\mathrm{g}_{M R I}$ of $0.76(\mathrm{SD}=0.05)$. Other groups have explored these and other MVF and AVF sensitive contrasts for g-ratio mapping in healthy white matter. These include a study of the effects of age and gender in a population of subjects aged 20 to 76 using qMT and NODDI (Cercignani et al. 2016b), studies of healthy adults using $\mathrm{MT}_{\text {sat }}$ and the TFD (Mohammadi et al., 2015) and MTV and DTI (Berman et al., 2017), and a study of healthy subjects using the ViSTa myelin water imaging technique and NODDI (Jung et al., 2016).

A variation of the g-ratio with age appears to be detectable with this methodology (Cercignani et al., 2016b). A variation with gender has not been seen, and if it exists in adolescence (Paus \& Toro, 2009), a study designed for sufficient statistical power at a precise age will be required to detect it. In addition to exploring the effect of age and gender, spatial variability of the g-ratio has been investigated. An elevated g-ratio at the splenium of the corpus callosum has been seen (Stikov et al. 2015b; Mohammadi et al., 2015). The splenium has been reported to contain axons of very large diameter (LaMantia \& Rakic, 1990), and these would be expected, due to the nonlinearity of the g-ratio (Hildebrand \& Hahn, 1978), to have relatively thinner myelin sheaths. Electron microscopy in the macaque (Stikov et al. 2015b) (see Fig. 11) confirms this; the "super-axons" dominate the aggregate g-ratio measure, which 

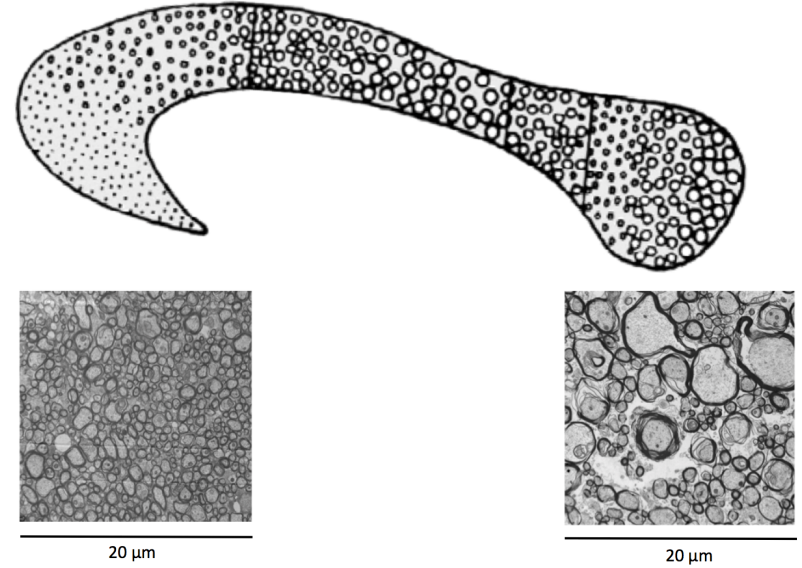

Figure 11: Existence of very large axons in the splenium of the corpus callosum. Top: drawing based on histology by Aboitiz et al. (reproduced from (Aboitiz \& Montiel 2003), showing large diameter at the splenium. Bottom: EM of the g-ratio in the cynamolgus macaque showing one sample from the genu (left) and one sample from the splenium (right). The splenium contains much larger diameter axons, and these will dominate the aggregate g-ratio measure, which was elevated in the splenium using both EM measurements and MRI of the same tissue shown here (Stikov et al. 2015b).

was seen to be elevated in the splenium using both EM measurements and MRI of the same tissue (Stikov et al., 2015b).

g-Ratio imaging has also been performed in the healthy human spinal cord (Duval et al., 2015), where there are considerable technical challenges, such as motion, susceptibility, and the need for significantly higher resolution than we have described for cerebral applications. Duval et al. acquired g-ratio data at $0.8 \mathrm{~mm} \times 0.8 \mathrm{~mm}$ inplane voxel size. This study used the CHARMED model of diffusion, more accessible on scanners with high gradient strength, on a CONNECTOM scanner. It used the MTV myelin marker. Of interest, the g-ratio was not found to vary significantly across white matter tracts in the spinal cord, while the diffusion metrics (restricted fraction, diffusivity of the hindered compartment, and axon diameter) and the MTV metric did vary across tracts. This is expected, as heterogeneity in packing and axon diameter is expected to be greater than heterogeneity of the g-ratio, and the g-ratio is also robust to partial voluming effects.

Multiple groups have studied the g-ratio in vivo in the developing brain (Dean et al., 2016; Melbourne et al., 2016). Axon growth outpaces myelination during development, and therefore a decreasing g-ratio is expected as myelination reaches maturity, as was seen in these studies.

\subsection{Multiple sclerosis}

Imaging the g-ratio in vivo in multiple sclerosis has been explored by several groups (Stikov et al., 2015b; Cercignani et al. 2015; Hori et al. 2016) and is of interest for several reasons. It can possibly help assess disease evolution, and can help monitor response to treatment. It has the potential to aid in the development of new therapies for remyelination. It can also help us understand which therapies might be more fruitful avenues of research. While currently available therapies are immunomodulatory or immunosuppressive in nature, several remyelinating therapies are in clinical trials. Preventing MS-related demyelination (via immunomodulatory therapies) is always preferred at earlier disease stages. Once demyelination has occurred, remyelinating therapies may help protect demyelinated (but not transected) axons from delayed degeneration due to the loss of trophic factors no longer received from myelin. Given that remyelination can occur only when demyelinated but still viable axons are present, interpretation of MRI markers of remyelination would be improved by including a marker of axonal integrity. Thus, the g-ratio framework would be useful in the evaluation of remyelinating therapies. In particular, dynamic changes of g-ratio over time could be measured within new and chronic lesions (as detected on conventional MRI) and the (temporally aligned) timecourses compared between treatment arms.

Despite the promise of imaging the g-ratio in vivo with MS, it is important to remember the pitfall of specificity and miscalibration of the myelin metric when interpreting g-ratio estimates in MS. In our own preliminary experience imaging MS, the relationship between the g-ratio in lesions and in NAWM appears to be complex. In section 3.3. we detailed our observations in one MS patient for whom we acquired a full qMT protocol. In a total of four subjects for whom we have acquired $\mathrm{MT}_{\text {sat }}$ data, we observed heterogeneity in the $\mathrm{g}_{M R I}$ values within and across subjects. The average ( \pm standard deviation) g-ratio in lesions compared to NAWM was not always higher than that in NAWM, with $\left(\mathrm{g}_{M R I}(\right.$ lesions $\left.), \mathrm{g}_{M R I}(\mathrm{NAWM})\right)=(0.80 \pm 0.07,0.76 \pm 0.05)$, $(0.72 \pm 0.05,0.77 \pm 0.03)$,

$(0.74 \pm 0.06,0.75 \pm 0.05),(0.75 \pm 0.08,0.78 \pm 0.04)$ for the four subjects. On average in these patients, $\mathrm{g}_{M R I}$ was 0.75 in lesions and 0.76 in NAWM. This variability of $\mathrm{g}_{M R I}$ in lesions compared to NAWM is consistent with preliminary findings from other groups using MT contrast for MVF (Cercignani et al., 2015), and could indicate that variable levels of non-myelin macromolecular content may confound the g-ratio metric. The g-ratio itself is not expected to be lower than in NAWM, but may appear so because other macromolecular content is confounding the MT measurements.

Further studies of MS are ongoing, including pediatric populations, optic neuritis, and studies investigating whether gadolinium enhancing lesions have a distinct g-ratio.

\subsection{Other potential applications}

g-Ratio imaging has potential to aid in the understanding and treatment of multiple other diseases. White matter abnormalities may underlie many developmental disorders. These include Pelizaeus Merzbacher disease and Sturge-Weber syndrome (Hori, 2016). An increased apparent g-ratio could result from axonal changes that occur with intact myelin (for example, axonal swelling due to infarction). g-Ratio differences have been seen in schizophrenia using electron microscopy (Uranova 
et al. 2001), and researchers hope to be able to study such changes in vivo in schizophrenia and other psychiatric disorders. Another potential application of g-ratio imaging is bridging the gap between microstructure and large-scale functional measures such as conduction delays. Adding a g-ratio measure to the human connectome could result in a framework for evaluating delays. Finally, g-ratio imaging offers the possibility to quantify plastic changes in myelin thickness due to learning and adaptation to injury or disease. MRI has been used to measure such changes, with the hypothesis that myelin thickness is changing (Reid et al. , 2016), but quantitative g-ratio measurement could help describe the neural changes in plasticity in more detail.

Of note, when examining the g-ratio compared to conduction velocity or functional metrics, linear correlation would not be expected to be a good statistic. The g-ratio has an optimal value, hence, both decreases and increases from this optimum would be expected to decrease function. In the future, assuming sufficient reproducibility, establishment of an age-dependent atlas of normal g-ratio values could help determine whether an individual lies within the normal range.

\subsection{Ex vivo g-ratio imaging}

One major application of ex vivo g-ratio imaging is validation of the technique for use in vivo, which should help elucidate the true promise of g-ratio imaging. Ex vivo validation has been performed, in order to investigate the g-ratio explicitly (Stikov et al. 2015b a; West et al. 2016a), or one or both of the individual metrics used to compute it (Duval et al. 2016b; Jelescu et al., 2016b; Jespersen et al., 2010, West et al., 2017, 2014, Schmierer et al., 2008, Scherrer et al., 2016, Sepehrband et al., 2015, Wood et al., 2016). These studies compare in vivo or ex vivo MRI metrics to electron microscopy, optical microscopy, myelin staining, immunohistochemistry, and coherent anti-Stokes Raman spectroscopy (CARS). While no microscopy technique is perfect, microscopy provides a reasonable validation for imaging techniques, taking into account the possibility for tissue shrinkage and distortion, limitations in contrast and resolution, and segmentation techniques (Zaimi et al. 2016).

Interpretation of findings of demyelinating models should take into account the particularities of the demyelinating challenge. Jelescu et al. have shown that the extra-axonal diffusivity perpendicular to axons correlates with the g-ratio in a cuprizone demyelinating model in mice (Jelescu et al., 2016b). This is probably driven by a fiber volume fraction decrease, because little axon loss would be expected in this model. In other words, the extra-axonal diffusivity is not specific to the g-ratio per se, but to the fiber volume fraction (i.e., size of the extracellular space), but these two quantities correlate highly in this particular case. Similarly, West et al. have shown a correlation between the discrepancy between $\mathrm{F}$ and MWF and the g-ratio in a knockout model in mice (West et al. 2014). This is probably a correlation with absolute myelin thickness, via exchange effects, as opposed to the g-ratio per se.

Ex vivo g-ratio imaging could also be of use for the study of neural tissue independent of validation of in vivo imaging. MRI is far more suited to imaging large samples of tissue than are other ex vivo imaging techniques, such as electron microscopy.

\section{Conclusion}

Computing a g-ratio metric is an effective way to interpret any combination of myelin-weighted and axon/fiber-weighted MR data. In this article, we have discussed the considerable promise of g-ratio imaging to help us understand disease, develop therapies, and monitor disease progression. Additionally, we have shown how the g-ratio framework can provide a window onto the study of normal brain variability, development, aging, plasticity, and functional dynamics. We have also explored the pitfalls of g-ratio imaging, which include MR artifacts, lack of specificity, low spatial resolution, and long acquisition times. Keeping these confounds in mind, it is clear that what we are currently measuring is an aggregate g-ratio weighted metric that is strongly dependent on the MRI markers used to compute it. The framework described in this article provides information on two quantities: the fiber density and the gratio (equivalently, the myelin and axon volume fractions), and attempts to decouple these two quantities to the best of the ability of our current imaging technology. To improve upon this description of the microstructure, one needs to fully decouple the g-ratio from the fiber density, and to provide precise and accurate measures of the myelin and the axon volume fractions. This task is at the frontier of microstructural MRI, showing the way for the future of multi-modal brain imaging.

\section{Acknowledgements}

The authors would like to thank Tomas Paus, Robert Dougherty, Eva Alonso-Ortiz, J.F. Cabana, Christine Tardif, Jessica Dubois, Dmitry Novikov, Ofer Pasternak, Atef Badji, Robert Brown, Masaaki Hori, and David Rudko for their insights and contributions to this work. This work was supported by grants from Campus Alberta Innovates, the Canadian Institutes for Health Research (GBP, FDN-143290, and JCA, FDN-143263), the Natural Science and Engineering Research Council of Canada (NS, 2016-06774, and JCA, 435897-2013), the Montreal Heart Institute Foundation, the Fonds de Recherche du Québec - Santé (JCA, 28826), the Quebec BioImaging Network (NS, 84360501), the Canada Research Chair in Quantitative Magnetic Resonance Imaging (JCA), and the Fonds de Recherche du Québec - Nature et Technologies (JCA, 2015-PR-182754).

\section{References}

\section{References}

Aboitiz, F., \& Montiel, J. (2003). One hundred million years of interhemispheric communication: the history of the corpus callosum. Brazilian Journal of Medical and Biological Research, 36. URL: http://dx.doi.org/10.1590/s0100-879x2003000400002 doi $10.1590 / \mathrm{s} 0100-879 \times 2003000400002$

Aboitiz, F., Scheibel, A. B., Fisher, R. S., \& Zaidel, E. (1992). Fiber composition of the human corpus callosum. Brain Research, 598, 143-153. URL: http://dx.doi.org/10.1016/0006-8993(92) $90178-\mathrm{C}$ doi $10.1016 / 0006-8993(92) 90178-\mathrm{c}$ 
Albert, M., Antel, J., Bruck, W., \& Stadelmann, C. (2007). Extensive cortical remyelination in patients with chronic multiple sclerosis. Brain Pathol, 17, 129-138. doi $10.1111 / j .1750-3639.2006 .00043 . x$

Alonso-Ortiz, E., Levesque, I. R., Paquin, R., \& Pike, G. B. (2016). Field inhomogeneity correction for gradient echo myelin water fraction imaging. Magn. Reson. Med., (pp. 49-57). URL: http://dx.doi.org/10. $1002 / \mathrm{mrm} .26334$ doi $10.1002 / \mathrm{mrm} .26334$

Andersson, J. L., Skare, S., \& Ashburner, J. (2003). How to correct susceptibility distortions in spin-echo echo-planar images: application to diffusion tensor imaging. NeuroImage, 20, 870-888. URL: http://dx.doi.org/10.1016/s1053-8119(03)00336-7 doi $10.1016 / \mathrm{s} 1053-8119$ (03) 00336-7

Assaf, Y., \& Basser, P. J. (2005). Composite hindered and restricted model of diffusion (CHARMED) MR imaging of the human brain . NeuroImage, 27, 48-58.

Assaf, Y., Blumenfeld-Katzir, T., Yovel, Y., \& Basser, P. J. (2008). AxCaliber: a method for measuring axon diameter distribution from diffusion MRI. Magn. Reson. Med., 59, 1347-1354. URL: http://dx.doi.org/10. $1002 / \mathrm{mrm} .21577$ doi $10.1002 / \mathrm{mrm} .21577$

Avram, A. V., Özarslan, E., Sarlls, J. E., \& Basser, P. J. (2013). In vivo detection of microscopic anisotropy using quadruple pulsed-field gradient (qPFG) diffusion MRI on a clinical scanner. NeuroImage, 64, 229-239. URL: http://dx.doi.org/10.1016/j.neuroimage. 2012.08 .048 doi $10.1016 / j . n e u r o i m a g e .2012 .08 .048$

Basser, P., Mattiello, J., \& Le Bihan, D. (1994). Estimation of the effective selfdiffusion tensor from the NMR spin echo. Journal of Magnetic Resonance, $103,247-254$.

Behrens, T. E., Berg, H. J., Jbabdi, S., Rushworth, M. F., \& Woolrich, M. W. (2007). Probabilistic diffusion tractography with multiple fibre orientations: What can we gain? Neurolmage., 34, 144-55.

Bells, S., Cercignani, M., Deoni, S., Assaf, Y., Pasternak, O., Evans, C. J., Leemans, A., \& Jones, D. K. (2011). Tractometry: Comprehensive multi-modal quantitative assessment of white matter along specific tracts. In ISMRM 2011 (p. 678).

Bengio, Y. (2009). Learning deep architectures for AI. Foundations and Trends in Machine Learning, 2, 1-127. URL: http://dx.doi.org/ $10.1561 / 2200000006$ doi $10.1561 / 2200000006$

Benninger, Y., Colognato, H., Thurnherr, T., Franklin, R. J. M., Leone, D. P., Atanasoski, S., Nave, K.-A., ffrench Constant, C., Suter, U., \& Relvas, J. B. (2006). 1-Integrin signaling mediates premyelinating oligodendrocyte survival but is not required for CNS myelination and remyelination. Journal of Neuroscience, 26, 7665-7673. URL: http://dx. doi.org/10.1523/jneurosci.0444-06.2006 doi 10.1523/ jneurosci.0444-06.2006

Berman, S., West, K. L., Does, M. D., Yeatman, J. D., \& Mezer, A. A. (2017). Evaluating g-ratio weighted changes in the corpus callosum as a function of age and sex. NeuroImage, URL:http://view.ncbi.nlm.nih. gov/pubmed/28673882

Berthold, C. H., Nilsson, I., \& Rydmark, M. (1983). Axon diameter and myelin sheath thickness in nerve fibres of the ventral spinal root of the seventh lumbar nerve of the adult and developing cat. J Anat, 136, 483-508.

Bjarnason, T. A., Vavasour, I. M., Chia, C. L., \& MacKay, A. L. (2005). Characterization of the NMR behavior of white matter in bovine brain. Magn. Reson. Med., 54, 1072-1081. URL:http://dx.doi.org/10.1002/ mrm.20680 doi $10.1002 / \mathrm{mrm} .20680$

Boudreau, M., Tardif, C. L., Stikov, N., Sled, J. G., Lee, W., \& Pike, G. B. (2017). B1 mapping for bias-correction in quantitative T1 imaging of the brain at $3 \mathrm{~T}$ using standard pulse sequences. Journal of magnetic resonance imaging, . URL: http://view. ncbi.nlm.nih. gov/pubmed/28301086

Bouhrara, M., Reiter, D. A., Celik, H., Fishbein, K. W., Kijowski, R., \& Spencer, R. G. (2016). Analysis of mcDESPOT- and CPMG-derived parameter estimates for two-component nonexchanging systems. Magn. Reson. Med., 75, 2406-2420. URL: http://dx.doi.org/10.1002/mrm. 25801 doi $10.1002 / \mathrm{mrm} .25801$

Bouyagoub, S., Dowell, N. G., Hurley, S. A., Wood, T. C., \& Cercignani, M. (2016). Overestimation of CSF fraction in NODDI: possible correction techniques and the effect on neurite density and orientation dispersion measures. In ISMRM 2016 (p. 0007).

Cabana, J.-F., Gu, Y., Boudreau, M., Levesque, I. R., Atchia, Y., Sled, J. G., Narayanan, S., Arnold, D. L., Pike, G. B., Cohen-Adad, J., Duval, T., Vuong,
M.-T., \& Stikov, N. (2015). Quantitative magnetization transfer imaging made easy with qMTLab: Software for data simulation, analysis, and visualization. Concepts Magn. Reson., 44A, 263-277. URL: http://dx. doi.org/10.1002/cmr.a.21357 doi 10.1002/cmr.a.21357

Callaghan, P. T., Godefroy, S., \& Ryland, B. N. (2003). Diffusionrelaxation correlation in simple pore structures. Journal of Magnetic Resonance, 162, 320-327. URL: http: //dx.doi.org/10.1016/s1090-7807(03)00056-9 doi $10.1016 /$ s1090-7807(03)00056-9

Campbell, J. S., Leppert, I. R., Boudreau, M., Narayanan, S., Duval, T., CohenAdad, J., Pike, G. B., \& Stikov, N. (2016). Caveats of miscalibration of myelin metrics for g-ratio imaging. In OHBM 2016 (p. 1804).

Campbell, J. S., Stikov, N., Dougherty, R. F., \& Pike, G. B. (2014). Combined NODDI and qMT for full-brain g-ratio mapping with complex subvoxel microstructure. In ISMRM 2014 (p. 393).

Campbell, J. S. W., \& Pike, G. B. (2013). Potential and limitations of diffusion MRI tractography for the study of language. Brain and Language, 131, 65-73. URL: http://dx.doi.org/10.1016/j. bandl.2013.06.007 doi 10.1016/j.bandl.2013.06.007

Cercignani, M., Giulietti, G., Dowell, N., Spano, B., Harrison, N., \& Bozzali, M. (2016a). A simple method to scale the macromolecular pool size ratio for computing the g-ratio in vivo. In ISMRM 2016 (p. 3369).

Cercignani, M., Giulietti, G., Dowell, N. G., Gabel, M., Broad, R., Leigh, P. N., Harrison, N. A., \& Bozzali, M. (2016b). Characterizing axonal myelination within the healthy population: a tract-by-tract mapping of effects of age and gender on the fiber g-ratio. Neurobiology of Aging, . URL: http://dx. doi.org/10.1016/j.neurobiolaging.2016.09.016 doi 10 . $1016 / j . n e u r o b i o l a g i n g .2016 .09 .016$

Cercignani, M., Giulietti, G., Spanó, B., \& Bozzali, M. (2015). Mapping the g-ratio within MS lesions. In ISMRM 2015 (p. 1402).

Chomiak, T., \& Hu, B. (2009). What is the optimal value of the g-ratio for myelinated fibers in the rat CNS? a theoretical approach. PLoS ONE, 4, e7754+. URL: http://dx.doi.org/10.1371/journal. pone.0007754 doi $10.1371 /$ journal.pone.0007754

Cohen-Adad, J. (2014). What can we learn from T2* maps of the cortex? NeuroImage, 93, 189-200. URL: http://dx.doi. org/10.1016/j.neuroimage.2013.01.023 doi $10.1016 / j$. neuroimage.2013.01.023

Daducci, A., Canales-Rodríguez, E. J., Zhang, H., Dyrby, T. B., Alexander, D. C., \& Thiran, J.-P. (2015). Accelerated microstructure imaging via convex optimization (AMICO) from diffusion MRI data. NeuroImage, 105, 32-44. URL: http://dx.doi.org/10.1016/j.neuroimage. 2014.10 .026 doi 10.1016/j.neuroimage.2014.10.026

De Santis, S., Barazany, D., Jones, D. K., \& Assaf, Y. (2016a). Resolving relaxometry and diffusion properties within the same voxel in the presence of crossing fibres by combining inversion recovery and diffusion-weighted acquisitions. Magn. Reson. Med., 75, 372-380. URL: http://dx.doi. org/10.1002/mrm.25644 doi $10.1002 / \mathrm{mrm} .25644$

De Santis, S., Jones, D. K., \& Roebroeck, A. (2016b). Including diffusion time dependence in the extra-axonal space improves in vivo estimates of axonal diameter and density in human white matter. NeuroImage, 130, 91-103. URL: http://dx.doi.org/10.1016/j.neuroimage. 2016.01 .047 doi $10.1016 / j . n e u r o i m a g e .2016 .01 .047$

Dean, D. C., O’Muircheartaigh, J., Dirks, H., Travers, B. G., Adluru, N., Alexander, A. L., \& Deoni, S. C. L. (2016). Mapping an index of the myelin g-ratio in infants using magnetic resonance imaging. Neurolmage, 132, 225-237. URL: http://dx.doi.org/10.1016/j.neuroimage. 2016.02 .040 doi $10.1016 / j . n e u r o i m a g e .2016 .02 .040$

Deoni, S. C. L., Rutt, B. K., Arun, T., Pierpaoli, C., \& Jones, D. K. (2008). Gleaning multicomponent $\mathrm{T} 1$ and $\mathrm{T} 2$ information from steady-state imaging data. Magn. Reson. Med., 60, 1372-1387. URL: http://dx.doi.org/ $10.1002 / \mathrm{mrm} .21704$ doi $10.1002 / \mathrm{mrm} .21704$

Descoteaux, M., Sidhu, J., Garyfallidis, E., Houde, J.-C., Neher, P., Stieltjes, B., \& Maier-Hein, K. H. (2016). False positive bundles in tractography. In ISMRM 2016 (p. 790).

Does, M. D., \& Gore, J. C. (2000). Rapid acquisition transverse relaxometric imaging. Journal of magnetic resonance, 147, 116-120. URL: http:// view.ncbi.nlm.nih.gov/pubmed/11042054

Du, J., Ma, G., Li, S., Carl, M., Szeverenyi, N. M., VandenBerg, S., CoreyBloom, J., \& Bydder, G. M. (2014). Ultrashort echo time (UTE) magnetic resonance imaging of the short $\mathrm{t} 2$ components in white matter of the 
brain using a clinical 3T scanner. NeuroImage, 87, 32-41. URL: http: // dx.doi.org/10.1016/j.neuroimage.2013.10.053 doil10. $1016 / j$.neuroimage.2013.10.053

Du, Y. P., Chu, R., Hwang, D., Brown, M. S., Kleinschmidt-DeMasters, B. K., Singel, D., \& Simon, J. H. (2007). Fast multislice mapping of the myelin water fraction using multicompartment analysis of T2* decay at 3T: a preliminary postmortem study. Magn. Reson. Med., 58, 865-870. URL: http: / / dx.doi.org/10.1002/mrm.21409 doi $10.1002 / \mathrm{mrm} .21409$

Dula, A. N., Gochberg, D. F., Valentine, H. L., Valentine, W. M., \& Does, M. D. (2010). Multiexponential T2, magnetization transfer, and quantitative histology in white matter tracts of rat spinal cord. Magn Reson Med, 63, 902-909. doi $10.1002 / \mathrm{mrm} .22267$

Duval, T., Lévy, S., Stikov, N., Campbell, J., Mezer, A., Witzel, T., Keil, B., Smith, V., Wald, L. L., Klawiter, E., \& Cohen-Adad, J. (2016a). g-Ratio weighted imaging of the human spinal cord in vivo. $\mathrm{Neu}$ rolmage, . URL: http://dx.doi.org/10.1016/j.neuroimage. 2016.09 .018 doi $10.1016 / j . n e u r o i m a g e .2016 .09 .018$

Duval, T., Levy, S., Stikov, N., Mezer, A., Witzel, T., Keil, B., Smith, V., Wald, L. L., Klawiter, E. C., \& Cohen-Adad, J. (2015). In vivo mapping of myelin g-ratio in the human spinal cord. In ISMRM 2015 (p. 5)

Duval, T., Perraud, B., Vuong, M.-T., Rios, N. L., Stikov, N., \& Cohen-Adad, J. (2016b). Validation of quantitative MRI metrics using full slice histology with automatic axon segmentation. In ISMRM 2016 (p. 396).

Edwards, L. J., Pine, K. J., Weiskopf, N., \& Mohammadi, S. (2016). NODDIDTI: biophysical parameters from DTI data. In ISMRM Workshop on Breaking the Barriers of Diffusion MRI (p. 8).

Ellerbrock, I., \& Mohammadi, S. (2017). Comparing in vivo MR g-ratio mapping methods: accuracy and precision at the group level. In ISMRM 2017 (p. 311)

Ferizi, U., Schneider, T., Witzel, T., Wald, L. L., Zhang, H., WheelerKingshott, C. A. M., \& Alexander, D. C. (2015). White matter compartment models for in vivo diffusion MRI at $300 \mathrm{mT} / \mathrm{m}$. NeuroImage, 118 , 468-483. URL: http://dx.doi.org/10.1016/j.neuroimage. 2015.06 .027 doi $10.1016 / j . n e u r o i m a g e .2015 .06 .027$

Fieremans, E., Burcaw, L. M., Lee, H.-H., Lemberskiy, G., Veraart, J., \& Novikov, D. S. (2016). In vivo observation and biophysical interpretation of time-dependent diffusion in human white matter. NeuroImage, 129, 414-427. URL: http://dx.doi.org/10.1016/j.neuroimage. 2016.01 .018 doi 10.1016/j.neuroimage.2016.01.018

Fieremans, E., De Deene, Y., Delputte, S., Özdemir, M. S., D’Asseler, Y., Vlassenbroeck, J., Deblaere, K., Achten, E., \& Lemahieu, I. (2008). Simulation and experimental verification of the diffusion in an anisotropic fiber phantom. Journal of Magnetic Resonance, 190, 189-199. URL: http: //dx.doi.org/10.1016/j.jmr.2007.10.014 doi 10.1016/ j.jmr.2007.10.014

Fieremans, E., Jensen, J. H., \& Helpern, J. A. (2011). White matter characterization with diffusional kurtosis imaging. NeuroImage, 58, 177-188. URL: http://dx.doi.org/10.1016/j.neuroimage. 2011.06 .006 doi $10.1016 / j . n e u r o i m a g e .2011 .06 .006$

Fram, E. K., Herfkens, R. J., Johnson, G. A., Glover, G. H., Karis, J. P., Shimakawa, A., Perkins, T. G., \& Pelc, N. J. (1987). Rapid calculation of T1 using variable flip angle gradient refocused imaging. Magnetic resonance imaging, 5, 201-208. URL: http://view.ncbi.nlm.nih. gov/pubmed/3626789

Garcia, M., Gloor, M., Radue, E.-W. W., Stippich, C. h., Wetzel, S. G., Scheffler, K., \& Bieri, O. (2012). Fast high-resolution brain imaging with balanced SSFP: Interpretation of quantitative magnetization transfer towards simple MTR. NeuroImage, 59, 202-211. URL: http://view.ncbi. nlm.nih.gov/pubmed/21820061

Gareau, P. J., Rutt, B. K., Karlik, S. J., \& Mitchell, J. R. (2000). Magnetization transfer and multicomponent $\mathrm{t} 2$ relaxation measurements with histopathologic correlation in an experimental model of MS. J. Magn. Reson. Imaging, 11, 586-595. URL: http://dx.doi.org/10. 1002/1522-2586(200006) 11:6\%3C586: :aid-jmri3\%3E3. $0 . \mathrm{Co} ; 2-\mathrm{v}$ doi $10.1002 / 1522-2586(200006) 11: 6 \backslash \div 3 \mathrm{C} 586:$ : aid-jmri3\ะ3E3.0.co;2-v

Ghosh, A., Alexander, D. C., \& Zhang, H. (2016). To be dispersed or not to be dispersed: A study using HCP data. In ISMRM 2016 (p. 792).

Girard, G., Fick, R., Descoteaux, M., Deriche, R., \& Wassermann, D. (2015) AxTract: Microstructure-Driven tractography based on the ensemble average propagator. IPMI 2015, 24, 675-686. URL: http://view.ncbi. nlm.nih.gov/pubmed/26221712

Glasser, M. F., \& Van Essen, D. C. (2011). Mapping human cortical areas in vivo based on myelin content as revealed by t1- and t2weighted MRI. J. Neurosci., 31, 11597-11616. URL: http://dx. doi.org/10.1523/jneurosci.2180-11.2011 doi 10.1523/ jneurosci.2180-11.2011

Grussu, F., Schneider, T., Zhang, H., Alexander, D. C., \& Wheeler-Kingshott, C. A. M. (2014). Single shell diffusion MRI NODDI with in vivo cervical cord data. In ISMRM 2014 (p. 1716).

Gupta, R., Rao, A., Kasiviswanathan, A., Chawla, S., Kumar, R., \& Venkatesan., R. (2003). Diffusion weighted EPI with magnetization transfer contrast. In ISMRM 2003 (p. 68).

Harkins, K. D., Dula, A. N., \& Does, M. D. (2012). Effect of intercompartmental water exchange on the apparent myelin water fraction in multiexponential T2 measurements of rat spinal cord. Magn. Reson. Med., 67, 793-800. URL: http://dx.doi.org/10.1002/mrm.23053 doi $10.1002 / \mathrm{mrm}$. 23053

Harkins, K. D., Xu, J., Dula, A. N., Li, K., Valentine, W. M., Gochberg, D. F., Gore, J. C., \& Does, M. D. (2016). The microstructural correlates of T1 in white matter. Magn. Reson. Med., 75, 1341-1345. URL: http://dx. doi.org/10.1002/mrm.25709 doi $10.1002 / \mathrm{mrm} .25709$

Helms, G., Dathe, H., Kallenberg, K., \& Dechent, P. (2008). High-resolution maps of magnetization transfer with inherent correction for RF inhomogeneity and T1 relaxation obtained from 3D FLASH MRI. Magn. Reson. Med., 60, 1396-1407. URL: http://dx.doi.org/10.1002/mrm.21732 doi $10.1002 / \mathrm{mrm} .21732$

Helms, G., Dathe, H., Kallenberg, K., \& Dechent, P. (2010). Erratum to: Helms, Dathe, Kallenberg and Dechent, High-resolution maps of magnetization transfer with inherent correction for RF inhomogeneity and T1 relaxation obtained from 3D FLASH MRI. Magn. Reson. Med. 2008; 60(6):13961407. Magn. Reson. Med., 64, 1856. URL: http://dx.doi.org/10. $1002 / \mathrm{mrm} .22607$ doi $10.1002 / \mathrm{mrm} .22607$

Hildebrand, C., \& Hahn, R. (1978). Relation between myelin sheath thickness and axon size in spinal cord white matter of some vertebrate species. $J$ Neurol Sci, 38, 421-434.

Hori, M. (2016). Toward clinically feasible acquisition protocol for g-ratio. In QBIN Workshop: Toward a super-big brain: promises and pitfalls of microstructural imaging.

Hori, M., Stikov, N., Nojiri, Y., Ryuji ad Tsurushima, Murata, K., Ishigame, K., Kamiya, K., Suzuki, Y., Kamagata, K., \& Aoki, S. (2016). Magnetic resonance myelin g-ratio mapping for the brain and cervical spinal cord: 10 minutes protocol for clinical application. In ISMRM 2016 (p. 3377).

Hutchinson, E. B., Avram, A., Komlosh, M., Irfanoglu, M. O., Barnett, A., Ozarslan, E., Schwerin, S., Radomski, K., Juliano, S., \& Pierpaoli, C. (2016). A systematic comparative study of dti and higher order diffusion models in brain fixed tissue. In ISMRM 2016 (p. 1048).

Hwang, D., Kim, D.-H., \& Du, Y. P. (2010). In vivo multi-slice mapping of myelin water content using $\mathrm{T} 2 *$ decay. NeuroImage, 52, 198-204. URL: http://dx.doi.org/10.1016/j.neuroimage. 2010.04 .023 doi $10.1016 / j . n e u r o i m a g e .2010 .04 .023$

Jelescu, I. O., Veraart, J., Adisetiyo, V., Milla, S. S., Novikov, D. S., \& Fieremans, E. (2015). One diffusion acquisition and different white matter models: How does microstructure change in human early development based on WMTI and NODDI? NeuroImage, 107, 242-256. URL: http://dx.doi.org/10.1016/j.neuroimage. 2014.12 .009 doi $10.1016 / j . n e u r o i m a g e .2014 .12 .009$

Jelescu, I. O., Veraart, J., Fieremans, E., \& Novikov, D. S. (2016a). Degeneracy in model parameter estimation for multi-compartmental diffusion in neuronal tissue. NMR Biomed., 29,33-47. URL: http://dx . doi .org/ $10.1002 / \mathrm{nbm} .3450$ doi $10.1002 / \mathrm{nbm} .3450$

Jelescu, I. O., Zurek, M., Winters, K. V., Veraart, J., Rajaratnam, A., Kim, N. S., Babb, J. S., Shepherd, T. M., Novikov, D. S., Kim, S. G., \& Fieremans, E. (2016b). In vivo quantification of demyelination and recovery using compartment-specific diffusion MRI metrics validated by electron microscopy. NeuroImage, 132, 104-114. URL: http://dx.doi. org/10.1016/j.neuroimage.2016.02.004 doi $10.1016 / j$. neuroimage.2016.02.004

Jespersen, S. N., Bjarkam, C. R., Nyengaard, J. R., Chakravarty, M. M., Hansen, B., Vosegaard, T., Østergaard, L., Yablonskiy, D., Nielsen, N. C. C., \& Vestergaard-Poulsen, P. (2010). Neurite density from magnetic resonance diffusion measurements at ultrahigh field: compar- 
ison with light microscopy and electron microscopy. Neurolmage, 49, 205-216. URL: http://dx.doi.org/10.1016/j.neuroimage. 2009.08 .053 doi $10.1016 / j$. neuroimage.2009.08.053

Jeurissen, B., Leemans, A., Tournier, J.-D., Jones, D. K., \& Sijbers, J. (2010). Estimating the number of fiber orientations in diffusion MRI voxels: a constrained spherical deconvolution study. In ISMRM 2010 (p. 573).

Jones, C. K., Xiang, Q.-S. S., Whittall, K. P., \& MacKay, A. L. (2004). Linear combination of multiecho data: short $\mathrm{t} 2$ component selection. Magn. Reson. Med., 51, 495-502. URL:/http://dx.doi.org/10.1002/mrm. 10713 doi $10.1002 / \mathrm{mrm} .10713$

Jung, W., Nam, Y., Zhang, H., \& Lee, J. (2016). Whole brain in-vivo g-ratio mapping using neurite orientation dispersion and density imaging (NODDI) and GRE myelin water imaging (GRE-MWI). In ISMRM 2016 (p. 1112).

Kaden, E., Kelm, N. D., Carson, R. P., Does, M. D., \& Alexander, D. C. (2016). Multi-compartment microscopic diffusion imaging. NeuroImage, 139, 346-359. URL:http://view.ncbi.nlm.nih.gov/pubmed/ 27282476

Graf von Keyserlingk, D., \& Schramm, U. (1984). Diameter of axons and thickness of myelin sheaths of the pyramidal tract fibres in the adult human medullary pyramid. Anatomischer Anzeiger, 157, 97-111. URL: http: //view.ncbi.nlm.nih.gov/pubmed/6507887

Kim, D., Kim, J. H., , \& Haldar, J. P. (2016). Diffusion-relaxation correlation spectroscopic imaging (DR-CSI): An enhanced approach to imaging microstructure. In ISMRM 2016 (p. 660)

Kiselev, V. G., \& Il'yasov, K. A. (2007). Is the biexponential diffusion biexponential? Magn. Reson. Med., 57, 464-469. URL: http://dx.doi. org $/ 10.1002 / \mathrm{mrm} .21164$ doi $10.1002 / \mathrm{mrm} .21164$

Kucharczyk, W., Macdonald, P. M., Stanisz, G. J., \& Henkelman, R. M. (1994). Relaxivity and magnetization transfer of white matter lipids at MR imaging: importance of cerebrosides and pH. Radiology, 192, 521-529. URL: http: //view.ncbi.nlm.nih.gov/pubmed/8029426

LaMantia, A. S., \& Rakic, P. (1990). Cytological and quantitative characteristics of four cerebral commissures in the rhesus monkey. The Journal of comparative neurology, 291, 520-537. URL: http://dx.doi.org/ $10.1002 / \mathrm{cne} .902910404$ doi $10.1002 / \mathrm{cne} .902910404$

Lampinen, B., Szczepankiewicz, F., Mrtensson, J., van Westen, D., Sundgren, P. C., \& Nilsson, M. (2017). Neurite density imaging versus imaging of microscopic anisotropy in diffusion MRI: A model comparison using spherical tensor encoding. NeuroImage, 147, 517-531. URL: http://dx.doi.org/10.1016/j.neuroimage. 2016.11.053 doi 10.1016/j.neuroimage.2016.11.053

Lampron, A., Larochelle, A., Laflamme, N., Préfontaine, P., Plante, M.M., Sánchez, M. G., Yong, V. W., Stys, P. K., Tremblay, M.-E., \& Rivest, S. (2015). Inefficient clearance of myelin debris by microglia impairs remyelinating processes. Journal of Experimental Medicine, 212, 481-495. URL: http://dx.doi.org/10.1084/jem.20141656 doi $10.1084 /$ jem. 20141656

Lankford, C. L., \& Does, M. D. (2013). On the inherent precision of mcDESPOT. Magn Reson Med, 69, 127-136. URL: http://dx.doi. org/10.1002/mrm.24241 doi $10.1002 / \mathrm{mrm} .24241$

Laule, C., Leung, E., Lis, D. K., Traboulsee, A. L., Paty, D. W., MacKay, A. L., \& Moore, G. R. (2006). Myelin water imaging in multiple sclerosis: quantitative correlations with histopathology. Multiple sclerosis, 12, 747-753. URL:http://view.ncbi.nlm.nih.gov/pubmed/17263002

Laule, C., Vavasour, I. M., Kolind, S. H., Li, D. K., Traboulsee, T. L., Moore, G. W., \& MacKay, A. L. (2007). Magnetic resonance imaging of myelin. Neurotherapeutics : the journal of the American Society for Experimental NeuroTherapeutics, 4, 460-484. URL: http:// dx.doi.org/10.1016/j.nurt.2007.05.004 doi 10.1016/j. nurt.2007.05.004

Lema, A., Bishop, C., Malik, O., Mattoscio, M., Ali, R., Nicholas, R., Muraro, P. A., Matthews, P. M., Waldman, A. D., \& Newbould, R. D. (2017). A comparison of magnetization transfer methods to assess brain and cervical cord microstructure in multiple sclerosis. J Neuroimaging, 27, 221-226. URL: http://dx.doi.org/10.1111/jon.12377 doi 10.1111/jon. 12377

Levesque, I., Sled, J. G., Narayanan, S., Santos, A. C., Brass, S. D., Francis, S. J., Arnold, D. L., \& Pike, G. B. (2005). The role of edema and demyelination in chronic T1 black holes: A quantitative magnetization transfer study. J. Magn. Reson. Imaging, 21, 103-110. URL: http://dx.doi.org/ $10.1002 /$ jmri.20231 doi $10.1002 /$ jmri.20231
Levesque, I. R., \& Pike, G. B. (2009). Characterizing healthy and diseased white matter using quantitative magnetization transfer and multicomponent T2 relaxometry: A unified view via a four-pool model. Magn. Reson. Med., 62, 1487-1496. URL:http://dx.doi.org/10.1002/mrm.22131 doi $10.1002 / \mathrm{mrm} .22131$

Liu, C., Li, W., Tong, K. A., Yeom, K. W., \& Kuzminski, S. (2015). Susceptibility-weighted imaging and quantitative susceptibility mapping in the brain. Journal of magnetic resonance imaging, 42, 23-41. URL: http://view.ncbi.nlm.nih.gov/pubmed/25270052

Lustig, M., Donoho, D., \& Pauly, J. M. (2007). Sparse MRI: The application of compressed sensing for rapid MR imaging. Magn. Reson. Med., 58, 11821195. URL: http://dx.doi.org/10.1002/mrm.21391 doi 10 . $1002 / \mathrm{mrm} .21391$

MacKay, A., Whittall, K., Adler, J., Li, D., Paty, D., \& Graeb, D. (1994). In vivo visualization of myelin water in brain by magnetic resonance. Magn Reson Med, 31, 673-677. URL:/http://view.ncbi.nlm.nih.gov/ pubmed/8057820

Magnollay, L., Grussu, F., Wheeler-Kingshott, C., Sethi, V., Zhang, H., Chard, D., Miller, D., \& Ciccarelli, O. (2014). An investigation of brain neurite density and dispersion in multiple sclerosis using single shell diffusion imaging. In ISMRM 2014 (p. 2048)

Maier-Hein, K., Neher, P., \& et al. (2016). Tractography-based connectomes are dominated by false-positive connections. bioRxiv, (pp. 084137+). URL: http://dx.doi.org/10.1101/084137 doi 10.1101/084137

Mangeat, G., Govindarajan, S. T., Mainero, C., \& Cohen-Adad, J. (2015). Multivariate combination of magnetization transfer, $\mathrm{T} 2 *$ and $\mathrm{B} 0$ orientation to study the myelo-architecture of the in vivo human cortex. NeuroImage, 119, 89-102. URL: http://dx.doi.org/10.1016/j.neuroimage. 2015.06 .033 doi $10.1016 / j . n e u r o i m a g e .2015 .06 .033$

Manning, A. P., Chang, K. L., MacKay, A. L., \& Michal, C. A. (2016). The physical mechanism of inhomogeneous magnetization transfer MRI. Journal of Magnetic Resonance, . URL: http://dx.doi.org/10.1016/ j.jmr.2016.11.013 doi 10.1016/j.jmr.2016.11.013

McLean, M., MacDonald, M., Lebel, R. M., Boudreau, M., \& Pike, G. B. (2017). Accelerated z-spectrum imaging. In ISMRM 2017 (p. 1205)

Melbourne, A., Eaton-Rosen, Z., Orasanu, E., Price, D., Bainbridge, A., Cardoso, M. J., Kendall, G. S., Robertson, N. J., Marlow, N., \& Ourselin, S. (2016). Longitudinal development in the preterm thalamus and posterior white matter: MRI correlations between diffusion weighted imaging and T2 relaxometry. Hum. Brain Mapp., (p. n/a). URL: http://dx.doi.org/ $10.1002 / \mathrm{hbm} .23188$ doi $10.1002 / \mathrm{hbm} .23188$

Mezer, A., Yeatman, J. D., Stikov, N., Kay, K. N., Cho, N.-J., Dougherty, R. F., Perry, M. L., Parvizi, J., Hua, L. H., Butts-Pauly, K., \& Wandell, B. A. (2013). Quantifying the local tissue volume and composition in individual brains with magnetic resonance imaging. Nat Med, 19, 1667-1672. URL: http://dx.doi.org/10.1038/nm.3390 doi $10.1038 / \mathrm{nm} .3390$

mincdiffusion (2013), . URL: http://www.bic.mni.mcgill.ca/ $\$\{\backslash$ sim\}\$ilana/diffusion/diffusion_tools.htm August 2013.

Mohammadi, S., Carey, D., Dick, F., Diedrichsen, J., Sereno, M. I., Reisert, M., Callaghan, M. F., \& Weiskopf, N. (2015). Whole-brain in-vivo measurements of the axonal g-ratio in a group of 37 healthy volunteers. Frontiers in neuroscience, 9. URL: http://dx.doi.org/10.3389/ fnins.2015.00441 doi 10.3389/fnins.2015.00441

Molina-Romero, M., Gomez, P. A., Sperl, J. I., Jones, D. K., Menzel, M. I., \& Menze1, B. H. (2016). Tissue microstructure characterisation through relaxometry and diffusion MRI using sparse component analysis. In ISMRM workshop: Breaking the Barriers of Diffusion MRI (p. 17).

Mollink, J., Kleinnijenhuis, M., Sotiropoulos, S. N., Cottaar, M., van Cappellen van Walsum, A.-M., Gamarallage, M. P., Ansorge, O., Jbabdi, S., \& Miller, K. L. (2016). Exploring fibre orientation dispersion in the corpus callosum: Comparison of diffusion mri, polarized light imaging and histology. In ISMRM 2016 (p. 795).

Mottershead, J. P., Schmierer, K., Clemence, M., Thornton, J. S., Scaravilli, F., Barker, G. J., Tofts, P. S., Newcombe, J., Cuzner, M. L., Ordidge, R. J., McDonald, W. I., \& Miller, D. H. (2003). High field MRI correlates of myelin content and axonal density in multiple sclerosis-a post-mortem study of the spinal cord. Journal of neurology, 250, 1293-1301. URL: http: //view.ncbi.nlm.nih.gov/pubmed/14648144

Nguyen, T. D., Deh, K., Monohan, E., Pandya, S., Spincemaille, P., Raj, A., 
Wang, Y., \& Gauthier, S. A. (2016). Feasibility and reproducibility of whole brain myelin water mapping in 4 minutes using fast acquisition with spiral trajectory and adiabatic t2prep (FAST-t2) at 3T. Magn. Reson. Med. 76, 456-465. URL: http://dx.doi.org/10.1002/mrm.25877 doi $10.1002 / \mathrm{mrm} .25877$

Ning, L., Westin, C.-F., \& Rathi, Y. (2016). Estimation of bounded and unbounded trajectories in diffusion MRI. Frontiers in Neuroscience, 10. URL: http://dx.doi.org/10.3389/fnins.2016.00129 doi $10.3389 /$ fnins.2016.00129

NODDI Matlab Toolbox (2013). http://cmic.cs.ucl.ac.uk/mig/index.php?n= Tutorial.NODDImatlab. May 2013.

Nossin-Manor, R., Card, D., Raybaud, C., Taylor, M. J., \& Sled, J. G. (2015). Cerebral maturation in the early preterm period-A magnetization transfer and diffusion tensor imaging study using voxel-based analysis. NeuroImage, 112, 30-42. URL:/http://view.ncbi.nlm.nih.gov/ pubmed/25731990

Novikov, D., \& Fieremans, E. (2012). Relating extracellular diffusivity to cell size distribution and packing density as applied to white matter. In ISMRM 2012 (p. 1829).

Novikov, D. S., Fieremans, E., Jensen, J. H., \& Helpern, J. A. (2011). Random walks with barriers. Nature Physics, 7, 508-514. URL: http://dx. doi . org/10.1038/nphys1936 doi $10.1038 /$ nphys1936

Novikov, D. S., Veraart, J., Jelescu, I. O., \& Fieremans, E. (2016). Mapping orientational and microstructural metrics of neuronal integrity with in vivo diffusion MRI. arXiv, (p. 1609.09144). URL: http: //arxiv.org/abs/ 1609.09144

Oh, J., Han, E. T., Pelletier, D., \& Nelson, S. J. (2006). Measurement of in vivo multi-component $\mathrm{T} 2$ relaxation times for brain tissue using multislice T2 prep at 1.5 and $3 \mathrm{~T}$. Magnetic resonance imaging, 24, 33 43. URL: http://dx.doi.org/10.1016/j.mri.2005.10.016 doi $10.1016 / j . m r i .2005 .10 .016$

Oh, S.-H., Bilello, M., Schindler, M., Markowitz, C. E., Detre, J. A., \& Lee, J. (2013). Direct visualization of short transverse relaxation time component (ViSTa). NeuroImage, 83, 485-492. URL: http://dx.doi. org/10.1016/j.neuroimage.2013.06.047 doi $10.1016 / j$. neuroimage.2013.06.047

Pampel, A., Müller, D. K., Anwander, A., Marschner, H., \& Möller, H. E. (2015). Orientation dependence of magnetization transfer parameters in human white matter. NeuroImage, 114, 136-146. URL: http://dx.doi . org/10.1016/j.neuroimage.2015.03.068 doi $10.1016 / j$. neuroimage.2015.03.068

Pasternak, O., Sochen, N., Gur, Y., Intrator, N., \& Assaf, Y. (2009). Free water elimination and mapping from diffusion MRI. Magn. Reson. Med., 62, 717-730. URL: http://dx.doi.org/10.1002/mrm.22055 doi $10.1002 / \mathrm{mrm} .22055$

Paus, T., \& Toro, R. (2009). Could sex differences in white matter be explained by g ratio? Frontiers in neuroanatomy, 3. URL: http://dx. doi.org/10.3389/neuro.05.014.2009 doi $10.3389 /$ neuro. 05.014 .2009

Perrin, J. S., Leonard, G., Perron, M., Pike, G. B., Pitiot, A., Richer, L., Veillette, S., Pausova, Z., \& Paus, T. (2009). Sex differences in the growth of white matter during adolescence. NeuroImage, 45, 1055-1066. doi $10.1016 / j$.neuroimage.2009.01.023

Pesaresi, M., Soon-Shiong, R., French, L., Kaplan, D. R., Miller, F. D., \& Paus, T. (2015). Axon diameter and axonal transport: In vivo and in vitro effects of androgens. NeuroImage, 115, 191-201. URL: http://dx.doi. org/10.1016/j.neuroimage.2015.04.048 doi $10.1016 / j$. neuroimage.2015.04.048

Prasloski, T., Rauscher, A., MacKay, A. L., Hodgson, M., Vavasour, I. M., Laule, C., \& Mädler, B. (2012). Rapid whole cerebrum myelin water imaging using a 3D GRASE sequence. NeuroImage, 63, 533-539. URL: http://dx.doi.org/10.1016/j.neuroimage. 2012.06.064 doi:10.1016/j.neuroimage.2012.06.064

Raffelt, D., Tournier, Rose, S., Ridgway, G. R., Henderson, R., Crozier, S., Salvado, O., \& Connelly, A. (2012). Apparent fibre density: A novel measure for the analysis of diffusion-weighted magnetic resonance images. NeuroImage, 59, 3976-3994. URL: http://dx.doi. org/10.1016/j.neuroimage.2011.10.045 doi $10.1016 / j$. neuroimage.2011.10.045

Ramani, A., Dalton, C., Miller, D. H., Tofts, P. S., \& Barker, G. J. (2002) Precise estimate of fundamental in-vivo MT parameters in human brain in clinically feasible times. Magnetic resonance imaging, 20,721-731. URL: http://view.ncbi.nlm.nih.gov/pubmed/12591568

Reid, L. B., Sale, M. V., Cunnington, R., Mattingley, J. B., \& Rose, S. E. (2016). Structural and functional brain changes following four weeks of unimanual motor training: evidence from fMRI-guided diffusion MRI tractography. bioRxiv, (pp. 088328+). URL: http://dx.doi.org/10.1101/ 088328 doi $10.1101 / 088328$

Reisert, M., Mader, I., Umarova, R., Maier, S., Tebartz van Elst, L., \& Kiselev, V. G. (2013). Fiber density estimation from single q-shell diffusion imaging by tensor divergence. NeuroImage, 77, 166-176. URL: http://dx.doi.org/10.1016/j.neuroimage. 2013.03.032 doi $10.1016 / j . n e u r o i m a g e .2013 .03 .032$

Rokem, A., Yeatman, J. D., Pestilli, F., Kay, K. N., Mezer, A., van der Walt, S., \& Wandell, B. A. (2015). Evaluating the accuracy of diffusion MRI models in white matter. PLoS ONE, 10, e0123272+. URL: http:// dx.doi.org/10.1371/journal.pone.0123272 doi $10.1371 /$ journal.pone.0123272

Ronen, I., Budde, M., Ercan, E., Annese, J., Techawiboonwong, A., \& Webb, A. (2014). Microstructural organization of axons in the human corpus callosum quantified by diffusion-weighted magnetic resonance spectroscopy of n-acetylaspartate and post-mortem histology. Brain structure \& function, 219, 1773-1785. URL: http://view.ncbi.nlm.nih. gov/pubmed/23794120

Rooney, W. D., Johnson, G., Li, X., Cohen, E. R., Kim, S.-G., Ugurbil, K., \& Springer, C. S. (2007). Magnetic field and tissue dependencies of human brain longitudinal $1 \mathrm{H} 2 \mathrm{O}$ relaxation in vivo. Magn. Reson. Med. 57, 308-318. URL: http://dx.doi.org/10.1002/mrm.21122 doi $10.1002 / \mathrm{mrm} .21122$

Rudko, D. A., Klassen, L. M., de Chickera, S. N., Gati, J. S., Dekaban, G. A., \& Menon, R. S. (2014). Origins of R2 orientation dependence in gray and white matter. PNAS, 111, E159-E167. URL: http://dx.doi.org/ $10.1073 /$ pnas.1306516111 doi $10.1073 /$ pnas.1306516111

Rushton, W. A. H. (1951). A theory of the effects of fibre size in medullated nerve. J Physiol, 115, 101-22.

Sati, P., van Gelderen, P., Silva, A. C., Reich, D. S., Merkle, H., de Zwart, J. A., \& Duyn, J. H. (2013). Micro-compartment specific t2* relaxation in the brain. Neurolmage, 77, 268-278. URL: http://dx.doi. org/10.1016/j.neuroimage.2013.03.005 doi $10.1016 / j$. neuroimage.2013.03.005

Scherrer, B., Jacobs, D., Taquet, M., des Rieux, A., Macq, B., Prabhu, S. P., \& Warfield, S. K. (2016). Measurement of restricted and hindered anisotropic diffusion tissue compartments in a rat model of wallerian degeneration. In ISMRM 2016 (p. 1087).

Schmierer, K., Scaravilli, F., Altmann, D. R., Barker, G. J., \& Miller, D. H. (2004). Magnetization transfer ratio and myelin in postmortem multiple sclerosis brain. Ann Neurol., 56, 407-415. URL: http: / / dx.doi .org/ 10.1002 /ana.20202 doi 10.1002 /ana.20202

Schmierer, K., Tozer, D. J., Scaravilli, F., Altmann, D. R., Barker, G. J., Tofts, P. S., \& Miller, D. H. (2007). Quantitative magnetization transfer imaging in postmortem multiple sclerosis brain. Journal of magnetic resonance imaging : JMRI, 26, 41-51. URL: http://dx.doi.org/10.1002/ jmri.20984 doi $10.1002 /$ jmri.20984

Schmierer, K., Wheeler-Kingshott, C. A., Tozer, D. J., Boulby, P. A., Parkes, H. G., Yousry, T. A., Scaravilli, F., Barker, G. J., Tofts, P. S., \& Miller, D. H. (2008). Quantitative magnetic resonance of postmortem multiple sclerosis brain before and after fixation. Magn Reson Med, 59, 268-277. URL: http://dx.doi.org/10.1002/mrm.21487 doi $10.1002 / \mathrm{mrm}$. 21487

Schröder, J. M., Bohl, J., \& von Bardeleben, U. (1988). Changes of the ratio between myelin thickness and axon diameter in human developing sural, femoral, ulnar, facial, and trochlear nerves. Acta Neuropathol, 76, 471-83.

Sepehrband, F., Clark, K. A., Ullmann, J. F. P., Kurniawan, N. D., Leanage, G., Reutens, D. C., \& Yang, Z. (2015). Brain tissue compartment density estimated using diffusion-weighted MRI yields tissue parameters consistent with histology. Hum. Brain Mapp., 36, 3687-3702. URL: http://dx. doi.org/10.1002/hbm.22872 doi 10.1002/hbm.22872

Setsompop, K., Gagoski, B. A., Polimeni, J. R., Witzel, T., Wedeen, V. J., \& Wald, L. L. (2012). Blipped-controlled aliasing in parallel imaging for simultaneous multislice echo planar imaging with reduced g-factor penalty. Magn. Reson. Med., 67, 1210-1224. URL: http://dx.doi.org/10. $1002 / \mathrm{mrm} .23097$ doi $10.1002 / \mathrm{mrm} .23097$ 
Shemesh, N., Barazany, D., Sadan, O., Bar, L., Zur, Y., Barhum, Y., Sochen, N., Offen, D., Assaf, Y., \& Cohen, Y. (2012). Mapping apparent eccentricity and residual ensemble anisotropy in the gray matter using angular double-pulsed-field-gradient MRI. Magn Reson Med, 68, 794-806. URL: http://dx.doi.org/10.1002/mrm.23300 doi $10.1002 / \mathrm{mrm}$. 23300

Sled, J. G., \& Pike, G. B. (2001). Quantitative imaging of magnetization transfer exchange and relaxation properties in vivo using MRI. Magn. Reson. Med., 46, 923-931. URL: http://dx. doi .org/10.1002/mrm. 1278 doi $10.1002 / \mathrm{mrm} .1278$.

Smith, S. M., Jenkinson, M., Woolrich, M. W., Beckmann, C. F., Behrens, T. E., Johansen-Berg, H., Bannister, P. R., De Luca, M., Drobnjak, I., Flitney, D. E., Niazy, R. K., Saunders, J., Vickers, J., Zhang, Y., De Stefano, N., Brady, J. M., \& Matthews, P. M. (2004). Advances in functional and structural MR image analysis and implementation as FSL. NeuroImage, 23 Suppl 1, S208-S219. URL: http://dx.doi. org/10.1016/j.neuroimage.2004.07.051 doi $10.1016 / j$. neuroimage.2004.07.051

Stanisz, G. J., Szafer, A., Wright, G. A., \& Henkelman, R. M. (1997). An analytical model of restricted diffusion in bovine optic nerve. Magn. Reson. Med., 37, 103-111. URL: http://view.ncbi.nlm.nih.gov/ pubmed/8978638

Stikov, N., Campbell, J. S., Stroh, T., Lavelée, M., Frey, S., Novek, J., Nuara, S., Ho, M.-K. K., Bedell, B. J., Dougherty, R. F., Leppert, I. R., Boudreau, M., Narayanan, S., Duval, T., Cohen-Adad, J., Picard, P.-A. A., Gasecka, A., Côté, D., \& Pike, G. B. (2015a). Quantitative analysis of the myelin g-ratio from electron microscopy images of the macaque corpus callosum. Data in brief, 4, 368-373. URL: http://dx.doi.org/10.1016/j. dib.2015.05.019 doi $10.1016 / j . d i b .2015 .05 .019$

Stikov, N., Campbell, J. S. W., Stroh, T., Lavelée, M., Frey, S., Novek, J., Nuara, S., Ho, M.-K., Bedell, B. J., Dougherty, R. F., Leppert, I. R., Boudreau, M., Narayanan, S., Duval, T., Cohen-Adad, J., Picard, P., Gasecka, A., Côté, D., \& Bruce Pike, G. (2015b). In vivo histology of the myelin g-ratio with magnetic resonance imaging. NeuroImage, . URL: http://dx.doi.org/10.1016/j.neuroimage. 2015.05.023 doi:10.1016/j.neuroimage.2015.05.023

Stikov, N., Perry, L. M., Mezer, A., Rykhlevskaia, E., Wandell, B. A., Pauly, J. M., \& Dougherty, R. F. (2011). Bound pool fractions complement diffusion measures to describe white matter micro and macrostructure. NeuroImage, 54, 1112-1121. URL: http://dx.doi. org/10.1016/j.neuroimage.2010.08.068 doi 10.1016/j. neuroimage.2010.08.068

Stollberger, R., \& Wach, P. (1996). Imaging of the active B1 field in vivo. Magn. Reson. Med., 35, 246-251. URL: http://dx.doi.org/10. $1002 / \mathrm{mrm} .1910350217$ doi $10.1002 / \mathrm{mrm} .1910350217$

Stüber, C., Morawski, M., Schäfer, A., Labadie, C., Wähnert, M., Leuze, C., Streicher, M., Barapatre, N., Reimann, K., Geyer, S., Spemann, D., \& Turner, R. (2014). Myelin and iron concentration in the human brain: a quantitative study of MRI contrast. NeuroImage, 93 Pt 1, 95-106. URL: http://view.ncbi.nlm.nih.gov/pubmed/24607447

Sveinsson, B., \& Dougherty, R. F. (2011). dSim: Simulating Diffusion in Biologically Realistic Tissue Models. Technical Report CNITR-001 Stanford Center for Cognitive and Neurobiological Imaging.

Szafer, A., Zhong, J., \& Gore, J. C. (1995). Theoretical model for water diffusion in tissues. Magn. Reson. Med., 33, 697-712. URL: http: //dx.doi.org/10.1002/mrm.1910330516 doi $10.1002 / \mathrm{mrm}$. 1910330516

Thiessen, J. D., Zhang, Y., Zhang, H., Wang, L., Buist, R., Del Bigio, M. R., Kong, J., Li, X.-M., \& Martin, M. (2013). Quantitative MRI and ultrastructural examination of the cuprizone mouse model of demyelination. NMR Biomed., 26, 1562-1581. URL: http://dx.doi.org/10. $1002 / \mathrm{nbm} .2992$ doi $10.1002 / \mathrm{nbm} .2992$

Uranova, N., Orlovskaya, D., Vikhreva, O., Zimina, I., Kolomeets, N., Vostrikov, V., \& Rachmanova, V. (2001). Electron microscopy of oligodendroglia in severe mental illness. Brain research bulletin, 55, 597-610. URL:http://view.ncbi.nlm.nih.gov/pubmed/11576756

Varma, G., Duhamel, G., de Bazelaire, C., \& Alsop, D. C. (2015). Magnetization transfer from inhomogeneously broadened lines: A potential marker for myelin. Magn Reson Med, 73, 614-622. URL: http://view. ncbi. nlm.nih.gov/pubmed/24604578

Vavasour, I. M., Laule, C., Li, D. K. B., Traboulsee, A. L., \& MacKay, A. L.
(2011). Is the magnetization transfer ratio a marker for myelin in multiple sclerosis? J. Magn. Reson. Imaging, 33, 710-718. URL: http://dx. doi.org/10.1002/jmri.22441 doi 10.1002/jmri.22441

Vidarsson, L., Conolly, S. M., Lim, K. O., Gold, G. E., \& Pauly, J. M. (2005). Echo time optimization for linear combination myelin imaging. Magn. Reson. Med., 53, 398-407. URL: http://dx.doi.org/10.1002/mrm. 20360 doi $10.1002 / \mathrm{mrm} .20360$

Volz, S., Nöth, U., Rotarska-Jagiela, A., \& Deichmann, R. (2010). A fast B1-mapping method for the correction and normalization of magnetization transfer ratio maps at 3 T. NeuroImage, 49, 3015-3026. URL: http: //view.ncbi.nlm.nih.gov/pubmed/19948229

Wang, Y., Wang, Q., Haldar, J. P., Yeh, F.-C., Xie, M., Sun, P., Tu, T.W., Trinkaus, K., Klein, R. S., Cross, A. H., \& Song, S.-K. (2011). Quantification of increased cellularity during inflammatory demyelination. Brain, 134, 3590-3601. URL: http://dx.doi.org/10.1093/ brain/awr307 doi $10.1093 /$ brain/awr307

Waxman, S. G. (1975). Integrative properties and design principles of axons. Int Rev Neurobiol, 18, 1-40.

Weiskopf, N., Suckling, J., Williams, G., Correia, M. M., Inkster, B., Tait, R., Ooi, C., Bullmore, E. T., \& Lutti, A. (2013). Quantitative multi-parameter mapping of R1, PD*, MT, and R2* at 3T: a multi-center validation. Frontiers in neuroscience, 7. URL: http://view.ncbi.nlm.nih.gov/ pubmed/23772204

West, K. L., Kelm, N. D., Carson, R. P., \& Does, M. D. (2016a). Quantitative assessment of g-ratio from MRI. In QBIN Workshop: Toward a super-big brain: promises and pitfalls of microstructural imaging (p. 28).

West, K. L., Kelm, N. D., Carson, R. P., \& Does, M. D. (2016b). A revised model for estimating g-ratio from MRI. NeuroImage, URL: http://dx.doi.org/10.1016/j.neuroimage.2015. 08.017 doi $10.1016 / j . n e u r o i m a g e .2015 .08 .017$

West, K. L., Kelm, N. D., Carson, R. P., Gochberg, D. F., Ess, K. C., \& Does, M. D. (2017). Myelin volume fraction imaging with MRI. NeuroImage, . URL: http://dx.doi.org/10.1016/j.neuroimage. 2016.12 .067 doi $10.1016 / j$. neuroimage.2016.12.067

West, K. L., Kelm, N. D., Gochberg, D. F., Carson, R. P., Ess, K. C., \& Does, M. D. (2014). Multiexponential T2 and quantitative magnetization transfer in rodent brain models of hypomyelination. In ISMRM 2014 (p. 2088).

White, N. S., Leergaard, T. B., D’Arceuil, H., Bjaalie, J. G., \& Dale, A. M. (2013). Probing tissue microstructure with restriction spectrum imaging: Histological and theoretical validation. Hum. Brain Mapp., 34, 327346. URL: http://dx.doi.org/10.1002/hbm.21454 doi 10 . $1002 / \mathrm{hbm} .21454$

Wilhelm, M. J., Ong, H. H., Wehrli, S. L., Li, C., Tsai, P.-H., Hackney, D. B., \& Wehrli, F. W. (2012). Direct magnetic resonance detection of myelin and prospects for quantitative imaging of myelin density. Proceedings of the National Academy of Sciences, 109, 9605-9610. URL: http:// dx.doi.org/10.1073/pnas.1115107109 doi 10.1073/pnas. 1115107109

Wolff, S., Eng, J., \& Balaban, R. (1991). Magnetization transfer contrast: Method for improving contrast in gradient-recalled-echo images. Radiology, 179, 133-137.

Wood, T. C., Simmons, C., Hurley, S. A., Vernon, A. C., Torres, J., Dell'Acqua, F., Williams, S. C. R., \& Cash, D. (2016). Whole-brain ex-vivo quantitative MRI of the cuprizone mouse model. PeerJ, 4, e2632+. URL: http: / / dx. doi.org/10.7717/peerj.2632 doi $10.7717 /$ peerj.2632

Wu, Z., He, H., Chen, Y., Chen, S., Liu, H., Du, Y. P., \& Zhong, J. (2016). Feasibility study of high resolution mapping for myelin water fraction and frequency shift using tissue susceptibility. In ISMRM 2016 (p. 31).

Xu, J., Li, H., Harkins, K. D., Jiang, X., Xie, J., Kang, H., Does, M. D., \& Gore, J. C. (2014). Mapping mean axon diameter and axonal volume fraction by MRI using temporal diffusion spectroscopy. Neurolmage, 103, 10-19. URL: http://dx.doi. org/10.1016/j.neuroimage.2014.09.006 doi $10.1016 / j$. neuroimage.2014.09.006

Yarnykh, V. L. (2002). Pulsed z-spectroscopic imaging of cross-relaxation parameters in tissues for human MRI: Theory and clinical applications. Magn. Reson. Med., 47, 929-939. URL: http://dx.doi.org/10.1002/ mrm.10120 doi $10.1002 / \mathrm{mrm} .10120$

Yarnykh, V. L. (2012). Fast macromolecular proton fraction mapping from a single off-resonance magnetization transfer measurement. Magn Reson Med, 68, 166-178. URL: http://view.ncbi.nlm.nih.gov/ 
pubmed/22190042

Yarnykh, V. L., \& Khodanovich (2015). Analytical method of correction of B1 errors in mapping of magnetization transfer ratio in highfield magnetic resonance tomography. Russian Physics Journal, 57, 1784-1788. URL: http://dx.doi.org/10.1007/s11182-015-0451-7 doi 10 . $1007 / \mathrm{s} 11182-015-0451-7$

Zaimi, A., Duval, T., Gasecka, A., Côté, D., Stikov, N., \& Cohen-Adad, J. (2016). AxonSeg: Open source software for axon and myelin segmentation and morphometric analysis. Frontiers in Neuroinformatics, 10. URL: http://dx.doi.org/10.3389/fninf.2016.00037 doi $10.3389 /$ fninf.2016.00037

Zhang, H., Hubbard, P. L., Parker, G. J. M., \& Alexander, D. C. (2011) Axon diameter mapping in the presence of orientation dispersion with diffusion MRI. NeuroImage, 56, 1301-1315. URL: http://dx.doi. org/10.1016/j.neuroimage.2011.01.084 doi $10.1016 / j$. neuroimage.2011.01.084

Zhang, H., Schneider, T., Wheeler-Kingshott, C. A., \& Alexander, D. C. (2012). NODDI: Practical in vivo neurite orientation dispersion and density imaging of the human brain. NeuroImage, 61, 1000-1016. URL: http:// dx.doi.org/10.1016/j.neuroimage.2012.03.072 doi 10 . 1016/j.neuroimage.2012.03.072

Zhou, W., Walsh, E., \& Laidlaw, D. (2013). DoubleAx: In-vivo axon measurement in the human corpus callosum using angular double-PFG MRI. In OHBM 2013 (p. 2222). 